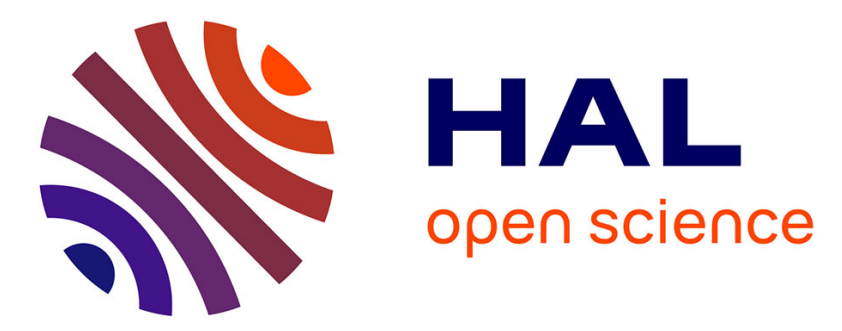

\title{
Self-similar perturbation near a corner: matching versus multiscale expansions for a model problem
}

\author{
Monique Dauge, Sébastien Tordeux, Grégory Vial
}

\section{To cite this version:}

Monique Dauge, Sébastien Tordeux, Grégory Vial. Self-similar perturbation near a corner: matching versus multiscale expansions for a model problem. Ari Laptev, Tamara Rozhkovskaya. Around the Research of Vladimir Maz'ya II, Partial Differential Equations., Springer, pp.95-134, 2010, International Mathematical Series, 10.1007/978-1-4419-1343-2_4 . hal-00124936v2

\section{HAL Id: hal-00124936 \\ https://hal.science/hal-00124936v2}

Submitted on 7 Jul 2009

HAL is a multi-disciplinary open access archive for the deposit and dissemination of scientific research documents, whether they are published or not. The documents may come from teaching and research institutions in France or abroad, or from public or private research centers.
L'archive ouverte pluridisciplinaire HAL, est destinée au dépôt et à la diffusion de documents scientifiques de niveau recherche, publiés ou non, émanant des établissements d'enseignement et de recherche français ou étrangers, des laboratoires publics ou privés. 


\title{
SELF-SIMILAR PERTURBATION NEAR A CORNER: MATCHING VERSUS MULTISCALE EXPANSIONS FOR A MODEL PROBLEM
}

\author{
MONIQUE DAUGE, SÉBASTIEN TORDEUX, AND GRÉGORY VIAL
}

\begin{abstract}
In this paper we consider the Laplace-Dirichlet equation in a polygonal domain perturbed at the small scale $\varepsilon$ near a vertex. We assume that this perturbation is self-similar, that is, derives from the same pattern for all relevant values of $\varepsilon$. We construct and validate asymptotic expansions of the solution in powers of $\varepsilon$ via two different techniques, namely the method of multiscale expansions and the method of matched asymptotic expansions. Then we show how the terms of each expansion can be split into a finite number of sub-terms in order to reconstruct the other expansion. Compared with the fairly general approach of Maz'ya, Nazarov and Plamenevskil relying on multiscale expansions, the novelty of our paper is the rigorous validation of the method of matched asymptotic expansions, and its comparison with the multiscale method. The consideration of a model problem allows to simplify the exposition of these rather complicated two techniques.
\end{abstract}

\section{CONTENTS}

1. Introduction 1

2. Notation, outline of results 3

3. Super-variational problems 6

4. Multi-Scale Expansion 11

5. Matching of asymptotic expansions 16

6. Comparison of the two expansions 22

7. Extensions and generalizations 23

8. Conclusion: A practical two-term expansion 29

Addresses $\quad 32$

\section{INTRODUCTION}

The perturbations under consideration concern the space domain, they have the same small scale $\varepsilon$ in every direction, and they are self-similar, which means that there exists a reference point $x_{0}$ and a pattern $\Omega$ such that the $\varepsilon$-perturbation is given by the set of point $x$ such that $\left(x-x_{0}\right) \varepsilon^{-1}$ belongs to $\Omega$. Although a local perturbation of the metric of a Riemannian manifold could be of interest as well, we only investigate in this paper the case when the perturbation involves the boundary of the domain. We are more particularly interested in the influence of corners, both in the unperturbed domain $\omega$ and in the perturbation pattern $\Omega$. 
An example of such a perturbation is given by rounded corners: Here the unperturbed domain $\omega$ is a domain with conical points, the perturbation pattern $\Omega$ is a smooth domain, and the limiting point $x_{0}$ of the perturbation is a conical point, see Fig.1. The fillets in material engineering precisely enter this framework.
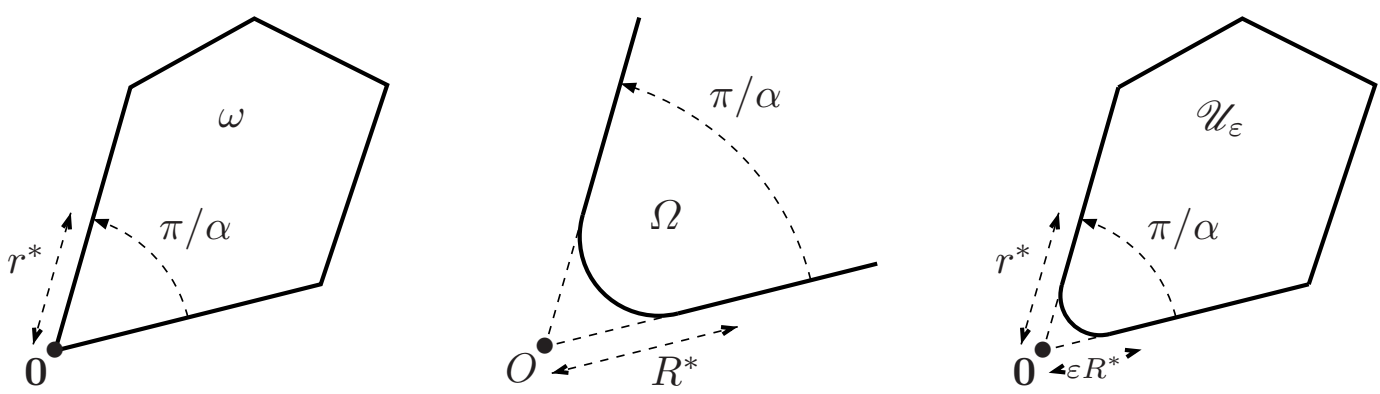

FIGURE 1. Rounded corner: Domains $\omega, \Omega$ and $\mathscr{U}_{\varepsilon}$.

The interesting and, at first glance puzzling, feature of such domains is the following: If one considers the solutions $u_{\varepsilon}$ of a common elliptic problem posed on such a family of domains $\mathscr{U}_{\varepsilon}$ with rounded corners, each solution $u_{\varepsilon}$ is smooth, but the sequence $u_{\varepsilon}$ converges as $\varepsilon \rightarrow 0$ to a limit solution in the corner domain $\omega$ which should contain singularities - we refer to the fundamental papers $[12,20]$ and to the books $[9,6,22,13]$ for the vast topic of singularities.

Conversely, one can consider smooth limiting domains $\omega$ and associated patterns $\Omega$ with corners, see Fig.2. In this case, the limiting solution is smooth and each solution $u_{\varepsilon}$ has corner singularities.
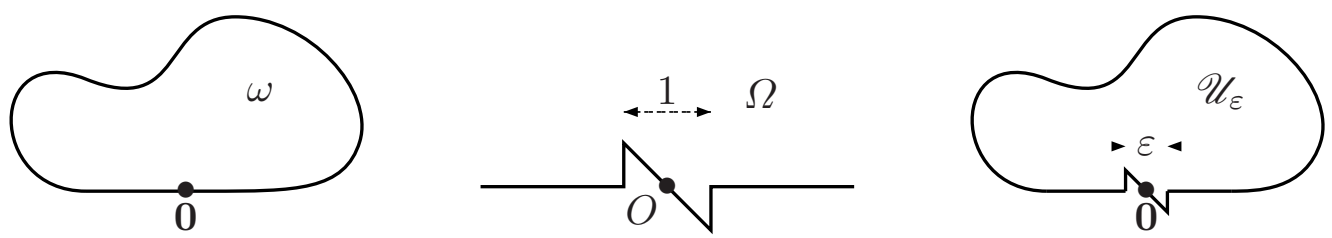

FIGURE 2. Corner perturbation originating from a smooth boundary point $(\alpha=1)$.

More generally, self-similar perturbations may include numerous different situations: Let us mention for example small cracks originating from a boundary point of the limiting domain, see Fig.5 p.30, and also small junctions between several connected components of $\omega$, see Fig.4 p.26.

For such singular perturbation problems, the method of matched asymptotic expansions is widely used. This method, spread by [25], consists in constructing two distinct complete expansions of the solution in different regions with different scalings, and to match them in an intermediate region. It has been used in [15] for the situation of Figure 1 (see also [10] for a general framework). Although intuitive, this method is difficult to justify rigorously, see $[24,11]$ for such a more recent justification in the case of thin slots. 
An alternative is given by the multi-scale expansion technique, consisting of a superposition of terms via cut-off functions, which involve different scales. An optimal rigorous error analysis can be performed for such a method. This analysis was performed V.G. Maz'ya and coauthors in $[17,18]$ and written in a very general framework in the monograph [19].

In this paper we mainly investigate, as a model case, the solutions $u_{\varepsilon}$ of the Dirichlet problem for the Laplace operator set on a family of plane self-similar domains $\mathscr{U}_{\varepsilon}$. For each fixed $\varepsilon$, the regularity properties of $u_{\varepsilon}$ can be very different from those of their limit $u_{0}$ (more or less regular, depending on different configurations, see Fig.1 and 2, respectively). An asymptotic expansion of $u_{\varepsilon}$ as $\varepsilon$ tends to 0 is the right way of understanding the mechanism of this transformation.

Our aim in this work is twofold

(i) Provide the complete constructions and validations of the two different expansions provided by the two methods of multi-scale expansion and matched asymptotic expansions for the same simple example, so that everything is made explicit and as clear as possible,

(ii) Compare the two expansions with each other, i.e. split each term of each expansion into sub-terms, and re-assemble them to reconstruct the terms of the other expansion.

Our paper is organized as follows. In Section 2, we define the families of self-similar domains and the problems under consideration, and next we provide an outline of our results, giving the structure of the first terms of both expansions. In Section 3 we state some preliminary results on limit problems in spaces with asymptotics, which we call "supervariational problems". Section 4 is devoted to the method of multi-scale expansion, like in $[4,26,3]$, where optimal remainder estimates are proved. In Section 5, we present the method of matched asymptotic expansions, with the construction of the terms and matching conditions, and, by the technique of $[24,11]$, the validation of the expansion by remainder estimates. Sections 4 and 5 may be read independently. We compare the expansions obtained by these two techniques in Section 6, providing formulas for the translation of the terms of each expansion into the terms of the other one. In Section 7 we mention how expansions can be generalized to other situations (more general domains, data, operators, etc...). We conclude in Section 8 with the definition of a "compound expansion" with the application to the study of the first singularity coefficient as $\varepsilon \rightarrow 0$.

\section{NotATiOn, OUTLINE OF RESUlts}

2.1. Self-similar perturbations. The families $\left(\mathscr{U}_{\varepsilon}\right)_{\varepsilon>0}$ under consideration are defined with the help of two domains, $\omega$ the limit (or unperturbed) domain, and $\Omega$ the pattern (or profile) of the perturbation. We denote by $x$ and $X$ the Cartesian coordinates in $\omega$ and $\Omega$, respectively, and by $\mathbf{0}$ and $O$ the corresponding origin of coordinates.

To simplify the exposition, we assume without restriction of the analysis that there is one perturbation and that the corresponding reference point $x_{0}$ coincides with the origin 0 . Indeed $\omega$ and $\Omega$ do not "live" in the same world. The $x$ coordinates are the slow variables and $X=\frac{x}{\varepsilon}$ are the fast variables.

The junction set. The connection between $\omega$ and $\Omega$ is realized by a plane sector $K$ with 
vertex at the origin. Let $\frac{\pi}{\alpha}$ be the opening of $K$, including the situations of a half-plane $(\alpha=1)$ or of a crack $\left(\alpha=\frac{1}{2}\right)$. Thus $K$ is a dilation invariant set and makes sense in both systems of coordinates $x$ and $X$.

We denote by $\mathscr{B}_{\rho}$ and $B_{\rho}$ the ball centered at the origin with radius $\rho$ in the $x$ and $X$ coordinates, respectively. Let $(r, \theta)$ and $(R, \theta)$ be polar coordinates corresponding to variables $x$ and $X$, respectively, and such that

$$
K=\left\{x \in \mathbb{R}^{2} ; r>0, \theta \in\left(0, \frac{\pi}{\alpha}\right)\right\}=\left\{X \in \mathbb{R}^{2} ; R>0, \theta \in\left(0, \frac{\pi}{\alpha}\right)\right\} .
$$

The limit domain. Let $\omega$ be a bounded domain of $\mathbb{R}^{2}$, containing the origin $\mathbf{0}$ in its boundary $\partial \omega$ and we assume that there exists $r^{*}>0$ such that

$$
\omega \cap \mathscr{B}_{r^{*}}=K \cap \mathscr{B}_{r^{*}} \text {. }
$$

The perturbing pattern. Let $\Omega$ be an unbounded domain of $\mathbb{R}^{2}$ such that there exists $R^{*}>0$ for which

$$
\Omega \cap \complement_{\mathbb{R}^{2}} B_{R^{*}}=K \cap \complement_{\mathbb{R}^{2}} B_{R^{*}}
$$

The perturbed domains. Let $\varepsilon_{0}$ such that $\varepsilon_{0} R^{*}=r^{*}$. For any $\varepsilon<\varepsilon_{0}$, $\mathscr{U}_{\varepsilon}$ denotes the bounded domain

$$
\mathscr{U}_{\varepsilon}=\left\{x \in \omega ;|x|>\varepsilon R^{*}\right\} \cup\left\{x \in \varepsilon \Omega ;|x|<r^{*}\right\} .
$$

The domain $\mathscr{U}_{\varepsilon}$ coincides with the limit domain $\omega$ except in an $\varepsilon$-neighborhood of the origin, where its shape is given by the $\varepsilon$-dilation of the domain $\Omega$, see Figures $1-2$. In the intermediate region $\varepsilon R^{*} \leqslant|x| \leqslant r^{*}, \mathscr{U}_{\varepsilon}$ coincides with $K$

$$
\mathscr{U}_{\varepsilon} \cap\left\{\varepsilon R^{*} \leqslant|x| \leqslant r^{*}\right\}=K \cap\left\{\varepsilon R^{*} \leqslant|x| \leqslant r^{*}\right\} .
$$

Note that $\Omega$ is the limit as $\varepsilon \rightarrow 0$ of the domain $\mathscr{U}_{\varepsilon} / \varepsilon$, whereas $\omega$ is the limit of $\mathscr{U}_{\varepsilon}$.

For the most part of this work, we do not assume any particular regularity for $\omega$ and $\Omega$, except the coincidence with the sector $K$ in the matching regions.

2.2. The Dirichlet problem and its singularities. As the simplest, and nevertheless typical, example of elliptic boundary value problem on a family $\left(\mathscr{U}_{\varepsilon}\right)$ of self-similarly perturbed domains, we consider the Laplace-Dirichlet problem. We are interested in asymptotic expansions with respect to $\varepsilon$ of the solution $u_{\varepsilon}$ of the problem

$$
\text { Find } u_{\varepsilon} \in \mathrm{H}_{0}^{1}\left(\mathscr{U}_{\varepsilon}\right) \text { such that }-\Delta u_{\varepsilon}=\left.f\right|_{\mathscr{U}_{\varepsilon}} \text { in } \mathscr{U}_{\varepsilon} \text {. }
$$

Here $f$ is a fixed function belonging to $\mathrm{L}^{2}\left(\mathbb{R}^{2}\right)$. We assume for simplicity ${ }^{1}$ that

$$
f \equiv 0 \text { in } \mathscr{B}_{r^{*}} \text {. }
$$

Thus the support of $f$ is contained in $\mathscr{U}_{\varepsilon} \backslash \mathscr{B}_{r^{*}}$, which coincides with $\omega \backslash \mathscr{B}_{r^{*}}$, hence independent of $\varepsilon$. Without risk of misunderstanding, we denote simply by $f$ the right hand side of (2.3).

When $\varepsilon$ tends to 0 , we expect the solution $u_{\varepsilon}$ of (2.3) to converge to the solution $u_{0}$ of the limit problem

$$
\text { Find } u_{0} \in \mathrm{H}_{0}^{1}(\omega) \text { such that }-\Delta u_{0}=f \text { in } \omega \text {. }
$$

\footnotetext{
${ }^{1}$ This assumption may be removed, see section 7.1 .
} 
In the following, we will derive the full asymptotic expansion of $u_{\varepsilon}$ into powers of $\varepsilon$. The nature of the terms in this expansion depends on the asymptotics as $r \rightarrow 0$ and $R \rightarrow \infty$ of solutions to the Dirichlet problem on the limit domain $\omega$ and the pattern domain $\Omega$, respectively.

Both asymptotics involve the singular functions of the Laplace-Dirichlet problem in the sector $K$, which solve the homogeneous problem

$$
\mathfrak{s}=0 \quad \text { on } \partial K \quad \text { and } \quad-\Delta \mathfrak{s}=0 \text { in } K .
$$

For the sector opening $\frac{\pi}{\alpha}$, a generating set for all solutions of (2.6) on the sector $K$ is given in polar coordinates $(\rho, \theta)$ by (see e.g. $[12,9])$

$$
\mathfrak{s}^{p \alpha}(\rho, \theta)=\rho^{p \alpha} \sin (p \alpha \theta), \quad \forall p \in \mathbb{Z}^{*} .
$$

2.3. Outline of results. As a result of our two methods of analysis, this expansion is described by two different formulas, the first terms of which we present now.

- The powers of $\varepsilon$ appearing in both formulas are the exponents $p \alpha$ of the singularities (2.7).

- The remainders in the following formulas are of the form $\mathcal{O}_{\mathrm{H}^{1}}\left(\varepsilon^{\alpha}\right)$, which means that their norms in $\mathrm{H}^{1}\left(\mathscr{U}_{\varepsilon}\right)$ are uniformly bounded by $C \varepsilon^{\alpha}$ as $\varepsilon \rightarrow 0$.

Multi-Scale Expansion: The method of Multi-Scale Expansion consists in looking for an expansion of $u_{\varepsilon}$ in powers of $\varepsilon$ with "coefficients" $v^{\alpha}(x)$ and $V^{\alpha}\left(\frac{x}{\varepsilon}\right)$ in slow and rapid variables respectively, and so that these terms are combined with each other by cut-off functions $\chi\left(\frac{x}{\varepsilon}\right)$ and $\psi(x)$ in rapid and slow variables respectively.

We choose a smooth function $X \mapsto \chi(X)$ which equals 1 except in a neighborhood of $O$ and another smooth function $x \mapsto \psi(x)$ with compact support and equal to 1 in a neighborhood of $\mathbf{0}$. The first step of the multi-scale expansion yields that

$$
u_{\varepsilon}=\chi\left(\frac{x}{\varepsilon}\right) v^{0}(x)+\mathcal{O}_{\mathrm{H}^{1}}\left(\varepsilon^{\alpha}\right),
$$

with $v^{0}=u_{0}$, which makes precise in what sense $u_{0}$ is the limit of $u_{\varepsilon}$. Thanks to the cut-off $\chi\left(\frac{x}{\varepsilon}\right)$, the term $\chi\left(\frac{x}{\varepsilon}\right) u^{0}(x)$ is well-defined on $\mathscr{U}_{\varepsilon}$ and is zero on the boundary $\mathscr{U}_{\varepsilon}$ in any configuration, $c f$. Figs 1 and 2 for instance. The next step of this method yields the two-term asymptotics

$$
u_{\varepsilon}=\chi\left(\frac{x}{\varepsilon}\right) v^{0}(x)+\psi(x) \varepsilon^{\alpha} V^{\alpha}\left(\frac{x}{\varepsilon}\right)+\mathcal{O}_{\mathrm{H}^{1}}\left(\varepsilon^{2 \alpha}\right),
$$

which proves in particular that the remainder in (2.8) is optimal. The general terms in the multi-scale expansion are $\chi\left(\frac{x}{\varepsilon}\right) v^{p \alpha}(x)$ and $\psi(x) V^{p \alpha}\left(\frac{x}{\varepsilon}\right)$, for integers $p=2, \ldots$, see Theorem 4.1 for an optimal estimate of remainders. The slow terms $v^{\lambda}(x), \lambda=2 \alpha, 3 \alpha, \ldots$, are also solution of variational problems in the limiting domain $\omega$, while the profiles $V^{\lambda}(X)$, $\lambda=\alpha, 2 \alpha, \ldots$ solve variational problems in the pattern $\Omega$.

The cut-off functions are used with a scale opposite to the associated terms of the asymptotic expansion. As a consequence, the transition region where both terms $v^{0}(x)$ and $V^{\lambda}\left(\frac{x}{\varepsilon}\right)$ contribute together to the asymptotics is the full domain (2.2) where $\mathscr{U}_{\varepsilon}$ coincides with the sector $K$. A wide range of problems can be treated like this, $c f$. [19, Ch.4]. The slow-rapid product Ansatz can also be compared with the homogenization and asymptotic expansions in periodic structures, see [23]. 
Matched Asymptotic Expansions: The method of Matched Asymptotic Expansions consists in constructing two different expansions (the inner and outer expansions) of $u_{\varepsilon}$ in rapid variables (near the perturbation) and slow variables (outside the perturbation). A priori, none of these expansions is unique or valid everywhere. They have to be matched inside an intermediate zone contained in the region (2.2). The method consists in matching the asymptotics as $X=\frac{x}{\varepsilon} \rightarrow \infty$ of the inner expansion with the asymptotics as $x \rightarrow \mathbf{0}$ of the outer expansion.

Following the approach of [8] or of [24] it is possible to construct an asymptotics of $u_{\varepsilon}$ valid everywhere with the help of a smooth cut-off function $\varphi$ at an intermediate scale $\varepsilon^{\delta}$, with a fixed $\delta \in(0,1)$. Let $\varphi$ be such that $\varphi(\rho)=0$ for $\rho \leqslant 1$ and $\varphi(\rho)=1$ for $\rho \geqslant 2$. By the method of Matched Asymptotic Expansions we find the following first terms, see Theorem 5.2,

$$
u_{\varepsilon}=\varphi\left(r / \varepsilon^{\delta}\right) u^{0}(x)+\left(1-\varphi\left(r / \varepsilon^{\delta}\right)\right) \varepsilon^{\alpha} U^{\alpha}\left(\frac{x}{\varepsilon}\right)+\mathcal{O}_{\mathrm{H}^{1}}\left(\varepsilon^{2 \alpha \beta}\right),
$$

with $\beta=\min \{\delta,(1-\delta)\}$. The remainders are optimized if we choose $\delta=\frac{1}{2}$. Here, again, the first term $u^{0}$ coincides with the limit $u_{0}$. The general asymptotics involve outer terms $u^{p \alpha}$, defined in $\omega$, and inner terms $U^{p \alpha}$ defined in the pattern $\Omega$. All of them are solution of what we call "super-variational problems", i.e. problems set in spaces larger than the variational spaces, see equations (3.9) and (3.17), and where standard formulations would have non-unique solutions.

Comparison: The terms $v^{2 \alpha}, V^{\alpha}, u^{2 \alpha}$ and $U^{\alpha}$ exchange with each other via two singular terms colinear to the singular functions $\mathfrak{s}^{\alpha}$ and $\mathfrak{s}^{-\alpha}, c f$. (2.7). There holds, see Theorem 6.1

$$
\left\{\begin{aligned}
U^{\alpha}(X) & =V^{\alpha}(X)+\chi(X) b_{1}^{0} \mathfrak{s}^{\alpha}(X), & & X \in \Omega, \\
u^{2 \alpha}(x) & =v^{2 \alpha}(x)+\psi(x) B_{1}^{1} \mathfrak{s}^{-\alpha}(x), & & x \in \omega .
\end{aligned}\right.
$$

Here $b_{1}^{0}$ and $B_{1}^{1}$ are the first coefficients of singularities for $v^{0}$ and $V^{\alpha}$, respectively. More generally, all terms of the matched inner and outer expansions can be reconstructed from the terms of the multi-scale expansion, and vice versa. The pros and contras of each method are

- The multiscale technique gives by construction a global approximation of the solution, with optimal estimates of the remainder, whereas twice as much terms are needed in the case of matched asymptotic expansions if one wants the same order for the remainder.

- The matched asymptotic expansions method builds outer and inner terms which are canonical, i.e. they do depend only on the domains $\omega$ and $\Omega$, and not on cut-off functions, as it is the case for the multiscale technique.

\section{SUPER-VARIATIONAL PROBLEMS}

In this section, we define the precise functional framework in which we will build the asymptotic expansions. The objects we define here are needed to derive rigorously both expansions.

All the terms in (2.8)-(2.10) appear as solutions of Dirichlet problems on $\omega$ or $\Omega$. We first recall their variational framework before considering their solutions in larger spaces. 
3.1. Variational problems. The variational space $V(\omega)$ for the Dirichlet problem on the bounded domain $\omega$ is $\mathrm{H}_{0}^{1}(\omega)$ and for $f$ in its dual space, the variational formulation is

$$
\left\{\begin{array}{l}
\text { Find } u \in V(\omega) \text { such that } \\
\quad \int_{\omega} \nabla u(x) \nabla v(x) d x=\int_{\omega} f(x) v(x) d x \quad \forall v \in V(\omega) .
\end{array}\right.
$$

Problem (3.1) has a unique solution. As a classical consequence of an angular Poincaré inequality, we find that the variational space is embedded into a weighted Sobolev space

$$
V(\omega)=\mathrm{H}_{0}^{1}(\omega) \subset \mathrm{W}_{0}^{1}(\omega):=\left\{u \in \mathrm{H}^{1}(\omega) ; r^{-1} u \in \mathrm{L}^{2}(\omega)\right\} .
$$

The variational space $V(\Omega)$ for the Dirichlet problem on the unbounded domain $\Omega$ is the weighted space

$$
V(\Omega)=\left\{U \in \mathrm{L}_{\text {loc }}^{2}(\Omega) ;\langle R\rangle^{-1} U \in \mathrm{L}^{2}(\Omega), \nabla U \in \mathrm{L}^{2}(\Omega),\left.U\right|_{\partial \Omega}=0\right\},
$$

where $\langle R\rangle=\sqrt{R^{2}+1}$. Then, for $f$ in the dual of $V(\Omega)$, the variational problem below has a unique solution

$$
\left\{\begin{array}{l}
\text { Find } U \in V(\Omega) \text { such that } \\
\qquad \int_{\Omega} \nabla U(X) \nabla V(X) d X=\int_{\Omega} f(X) V(X) d X \quad \forall V \in V(\Omega) .
\end{array}\right.
$$

One can refer for example to [3] for more details.

3.2. Super-variational problems in $\omega$. Behavior at the origin. First, we introduce some functional spaces to specify the behavior near the origin.

Definition 3.1. (i) Let $V_{\mathrm{loc}, 0}(\omega)$ be the space of distributions

$$
V_{\text {loc }, 0}(\omega)=\left\{u \in \mathscr{D}^{\prime}(\omega) ; \varphi u \in \mathrm{H}_{0}^{1}(\omega), \forall \varphi \in \mathscr{D}\left(\mathbb{R}^{2} \backslash\{\mathbf{0}\}\right)\right\} .
$$

(ii) For $m \in \mathbb{N}$ and $s \in \mathbb{R}$ let $\mathrm{W}_{s}^{m}(\omega)$ be the weighted Sobolev space

$$
\mathrm{W}_{s}^{m}(\omega)=\left\{u \in \mathscr{D}^{\prime}(\omega) ; r^{|\beta|-s-1} \partial_{x}^{\beta} u \in \mathrm{L}^{2}(\omega), \quad \forall \beta,|\beta| \leqslant m\right\} .
$$

Then, we particularize the meaning of $\mathcal{O}\left(r^{s}\right)$ as follows:

Notation 3.2. For $s \in \mathbb{R}$, the function $u: \omega \rightarrow \mathbb{R}$ is said to be a $\mathcal{O}_{r \rightarrow 0}\left(r^{s}\right)$ and we write $u=\mathcal{O}_{r \rightarrow 0}\left(r^{s}\right)$ if there exists a neighborhood $\mathscr{V}$ of $\mathbf{0}$ in $\mathbb{R}^{2}$ such that

$$
\forall m, n \in \mathbb{N}, \quad \exists C>0, \quad\left|r^{m} \partial_{r}^{m} \partial_{\theta}^{n} u\right| \leqslant C r^{s} \text { in } \omega \cap \mathscr{V} .
$$

Combining the change of variables $x \mapsto(t=\log r, \theta)$ with Sobolev embeddings, we prove:

$$
u \in \mathrm{W}_{s}^{m}(\omega \cap \mathscr{V}), \forall m \in \mathbb{N} \quad \Longrightarrow \quad u=\mathcal{O}_{r \rightarrow 0}\left(r^{s}\right)
$$

Note that the converse implication is not true: the function $x \mapsto r^{s}$ is a $\mathcal{O}_{r \rightarrow 0}\left(r^{s}\right)$, but does not even belong to $\mathrm{W}_{s}^{0}(\omega \cap \mathscr{V})$.

For functions harmonic in a neighborhood of the corner $\mathbf{0}$, there holds: 
Lemma 3.3. Let $u \in V_{\mathrm{loc}, 0}(\omega)$ such that $\Delta u=0$ in $\omega \cap \mathscr{V}$ for a neighborhood $\mathscr{V}$ of $\mathbf{0}$. Then for any real number $s$, we have the implication

$$
u \in \mathrm{W}_{s}^{1}(\omega) \Longrightarrow u=\mathcal{O}_{r \rightarrow 0}\left(r^{s}\right) .
$$

Proof. Let $u \in \mathrm{W}_{s}^{1}(\omega)$ satisfying the assumptions of the lemma. Let $\rho^{\prime} \in\left(0, r^{*}\right]$ such that the finite sector $K_{\rho^{\prime}}:=\omega \cap \mathscr{B}_{\rho^{\prime}}$ is contained in $\mathscr{V}$. Let $\rho \in\left(0, \rho^{\prime}\right)$, and $m \in \mathbb{N}$ be fixed. Let us prove that $u$ belongs to $\mathrm{W}_{s}^{m+2}\left(K_{\rho}\right)$, where $K_{\rho}=\omega \cap \mathscr{B}_{\rho}$.

For this, we consider two sectorial annuli, $\mathscr{A}$ and $\mathscr{A}^{\prime}$, defined as

$$
\mathscr{A}=\left\{x \in \omega \quad \rho_{0}<|x|<\rho\right\} \quad \text { and } \quad \mathscr{A}^{\prime}=\left\{x \in \omega \rho_{0}^{\prime}<|x|<\rho^{\prime}\right\},
$$

with $\rho_{0}^{\prime}<\rho_{0}<\rho / 2$, whence $\mathscr{A} \subset \mathscr{A}^{\prime}$. A standard local elliptic estimate reads, for $u$ satisfying $u \in \mathrm{W}_{s}^{1}\left(K_{\rho^{\prime}}\right), \Delta u \in \mathrm{W}_{s+2}^{m}\left(K_{\rho^{\prime}}\right)$, and $u=0$ on $\partial \omega \cap \mathscr{B}_{\rho^{\prime}}-$ see [1],

$$
\|u\|_{\mathrm{H}^{m+2}(\mathscr{A})} \leqslant C\left(\|\Delta u\|_{\mathrm{H}^{m}\left(\mathscr{A}^{\prime}\right)}+\|u\|_{\mathrm{H}^{1}\left(\mathscr{A}^{\prime}\right)}\right) .
$$

Applying this estimate to the functions $u_{k}(x)=u\left(2^{-k} x\right)$ and summing up over $k$ the obtained inequalities (multiplied by $2^{-s k}$ ), we get the following estimate from dyadic partition equivalence

$$
\|u\|_{\mathrm{W}_{s}^{m+2}\left(K_{\rho}\right)} \leqslant C\left(\|\Delta u\|_{\mathrm{W}_{s+2}^{m}\left(K_{\rho^{\prime}}\right)}+\|u\|_{\mathrm{W}_{s}^{1}\left(K_{\rho^{\prime}}\right)}\right) .
$$

The conclusion then follows from (3.5).

We can now state about the solvability of super-variational problems on $\omega$, that is, in spaces containing some of the dual singular functions $\mathfrak{s}^{-p \alpha}$ for $p \geqslant 1$ : If we know the dual singular part of a function $u \in V_{\mathrm{loc}, 0}(\omega)$ and its Laplacian $\Delta u$, then this function is uniquely defined.

Proposition 3.4. For any data $f \in \mathrm{H}^{-1}(\omega), f \equiv 0$ in a neighborhood of 0 , and any finite sequence $\left(a_{p}\right)_{1 \leqslant p \leqslant P}$ of real numbers, there exists a unique solution $u$ to the "supervariational problem"

$$
\left\{\begin{aligned}
\text { Find } u & \in V_{\mathrm{loc}, 0}(\omega) \text { such that } \\
-\Delta u & =f \text { in } \omega \text { and } u-\sum_{p=1}^{P} a_{p} \mathfrak{s}^{-p \alpha}=\mathcal{O}_{r \rightarrow 0}(1) .
\end{aligned}\right.
$$

Remark 3.5. If the sequence of coefficients $\left(a_{p}\right)_{p}$ is empty, the problem (3.9) is nothing but the variational problem (3.1).

Proof. Let the smooth cut-off function $\psi$ satisfy $\psi(x)=1$ for $|x|<r^{*} / 2$ and $\psi(x)=0$ for $|x|>r^{*}$. We set $v=\psi \sum_{p} a_{p} \mathfrak{s}^{-p \alpha}$, which obviously satisfies

$$
v \in V_{\mathrm{loc}, 0}(\omega), \quad \text { and } \quad \Delta v=0 \text { in } \omega \cap \mathscr{B}_{r^{*} / 2} .
$$

Hence, the problem to find $w$ such that $-\Delta w=f+\Delta v$ in $\omega$ admits a unique variational solution $w \in V(\omega)=\mathrm{H}_{0}^{1}(\omega)$. Moreover, (3.2) gives that $w$ belongs to $\mathrm{W}_{0}^{1}(\omega)$, and by localization near point $\mathbf{0}, w$ is a $\mathcal{O}_{r \rightarrow 0}(1)$ thanks to (3.6); the function $u=w+v$ meets then the requirements. 
On the other hand, every solution of the Laplace-Dirichlet equation can be expanded near the corner point $\mathbf{0}$ in terms of the singular functions, compare with the results in e.g. $[12,20,21,9]$.

Proposition 3.6. Let $s \geqslant 0$ be a real number. We define $P$ as the integer part of $s / \alpha$. For any $u \in V_{\mathrm{loc}, 0}(\omega)$ for which there is a neighborhood $\mathscr{V}$ of $\mathbf{0}$ such that

$$
\Delta u=0 \text { in } \omega \cap \mathscr{V} \text { and } u=\mathcal{O}_{r \rightarrow 0}\left(r^{-s}\right) \text {, }
$$

there exist a unique finite sequence $\left(a_{p}\right)_{1 \leqslant p \leqslant P}$ and a unique sequence $\left(b_{p}\right)_{p \in \mathbb{N}^{*}}$ (generically infinite) such that for all $N \in \mathbb{N}^{*}$

$$
u(x)=\sum_{p=1}^{P} a_{p} \mathfrak{s}^{-p \alpha}(r, \theta)+\sum_{p=1}^{N} b_{p} \mathfrak{s}^{p \alpha}(r, \theta)+\mathcal{O}_{r \rightarrow 0}\left(r^{(N+1) \alpha}\right) .
$$

Notation 3.7. In the situation of Proposition 3.6, we write

$$
u(x) \underset{r \rightarrow 0}{\simeq} \sum_{p=1}^{P} a_{p} \mathfrak{s}^{-p \alpha}(r, \theta)+\sum_{p=1}^{\infty} b_{p} \mathfrak{s}^{p \alpha}(r, \theta) .
$$

Proof. One can prove this lemma using the Mellin transform, see [12]. In the particular case we are interested in, an argument based on separation of variables via angular Fourier series also leads to the result.

In accordance with the literature on corner asymptotics $[21,7,5]$ we can call the sum $\sum a_{p} \mathfrak{s}^{-p \alpha}$ the dual singular part of $u$, whereas $\sum b_{p} \mathfrak{s}^{p \alpha}$ represents the asymptotics of the variational part of $u$ and can be called primal singular part of $u$.

In the particular case of an opening angle equal to $\pi$, i.e. $\alpha=1$, the asymptotics of the variational part contains polynomials only - it is a Taylor expansion, but the dual singular part is actually singular. More generally, if the opening angle has the form $\frac{\pi}{n}$ with a positive integer $n$, i.e. $\alpha=n$, the asymptotics of the variational part is polynomial and can be regarded as regular.

3.3. Super-variational problems in $\Omega$. Behavior at infinity. We give for the pattern domain $\Omega$ similar definitions and results as in the previous section, $r \rightarrow 0$ being replaced with $R \rightarrow+\infty$.

Definition 3.8. (i) Let $V_{\mathrm{loc}, \infty}(\Omega)$ be the space of distributions

$$
V_{\text {loc }, \infty}(\Omega)=\left\{U \in \mathscr{D}^{\prime}(\Omega) ; \varphi U \in \mathrm{H}_{0}^{1}(\Omega), \forall \varphi \in \mathscr{D}\left(\mathbb{R}^{2}\right)\right\} \text {. }
$$

(ii) For $m \in \mathbb{N}$ and $s \in \mathbb{R}$ let $\mathrm{W}_{s}^{m}(\Omega)$ be the weighted Sobolev space

$$
\mathrm{W}_{s}^{m}(\Omega)=\left\{U \in \mathscr{D}^{\prime}(\Omega) ;\langle R\rangle^{|\beta|-s-1} \partial_{X}^{\beta} U \in \mathrm{L}^{2}(\Omega), \quad \forall \beta,|\beta| \leqslant m\right\},
$$

where $\langle R\rangle=\sqrt{R^{2}+1}$.

In the following, we shall say that $W$ is a neighborhood of infinity if there exists a ball $B_{R}$ of radius $R$ such that

$$
\complement_{\mathbb{R}^{2}} B_{R} \subset W .
$$

We introduce, similarly to Notation 3.2 
Notation 3.9. For $s \in \mathbb{R}$, the function $U: \Omega \rightarrow \mathbb{R}$ is said to be a $\mathcal{O}_{R \rightarrow \infty}\left(R^{s}\right)$ and we write $U=\mathcal{O}_{R \rightarrow \infty}\left(R^{s}\right)$ if there exists a neighborhood $W$ of infinity such that

$$
\forall m, n \in \mathbb{N}, \quad \exists C>0, \quad\left|R^{m} \partial_{R}^{m} \partial_{\theta}^{n} U(R, \theta)\right| \leqslant C R^{s} \quad \text { in } \Omega \cap W .
$$

We have the implication

$$
u \in \mathrm{W}_{s}^{m}(\Omega \cap W), \forall m \in \mathbb{N} \quad \Longrightarrow \quad u=\mathcal{O}_{R \rightarrow \infty}\left(R^{s}\right) .
$$

Thanks to a similar shift result as for Lemma 3.3, we get

Lemma 3.10. Let $U \in V_{\mathrm{loc}, \infty}(\Omega)$ such that $\Delta U=0$ in $\Omega \cap W$ for a neighborhood $W$ of infinity. Then for any real number $s$, we have the implication

$$
U \in \mathrm{W}_{s}^{1}(\Omega) \quad \Longrightarrow \quad U=\mathcal{O}_{R \rightarrow \infty}\left(R^{s}\right) .
$$

The following two propositions are the counterparts of Propositions 3.4 and 3.6. The dual singular functions at infinity in $\Omega$ are now the $\mathfrak{s}^{p \alpha}$ for positive integers $p$.

Proposition 3.11. For any $F \in \mathrm{H}^{-1}(\Omega)$ with compact support in $\bar{\Omega}$ and any finite sequence $\left(A_{p}\right)_{1 \leqslant p \leqslant P}$ of real numbers, there exists a unique solution $U$ to the "super-variational problem”

$$
\left\{\begin{aligned}
\text { Find } U & \in V_{\mathrm{loc}, \infty}(\Omega) \text { such that } \\
-\Delta U & =F \text { in } \Omega \text { and } U-\sum_{p=1}^{P} A_{p} \mathfrak{s}^{p \alpha}=\mathcal{O}_{R \rightarrow \infty}(1) .
\end{aligned}\right.
$$

Proof. It is very similar to Proposition 3.4, the suitable variational space being here $V(\Omega)=$ $\mathrm{W}_{0}^{1}(\Omega)$.

Remark 3.12. If the sequence of coefficients $\left(A_{p}\right)$ is empty, the problem (3.17) is nothing but the variational problem (3.4).

Proposition 3.13. Let $s \geqslant 0$ be a real number. We define $P$ as the integer part of $s / \alpha$. For any $U \in V_{\mathrm{loc}, \infty}(\Omega)$ for which there is a neighborhood $W$ of infinity such that

$$
\Delta U=0 \text { in } \Omega \cap W \text { and } U=\mathcal{O}_{R \rightarrow \infty}\left(R^{s}\right),
$$

there exist a unique finite sequence $\left(A_{p}\right)_{1 \leqslant p \leqslant P}$ and a unique sequence $\left(B_{p}\right)_{p \in \mathbb{N}^{*}}$ (generically infinite) such that for all $N \in \mathbb{N}^{*}$

$$
U(X)=\sum_{p=1}^{P} A_{p} \mathfrak{s}^{p \alpha}(R, \theta)+\sum_{p=1}^{N} B_{p} \mathfrak{s}^{-p \alpha}(R, \theta)+\mathcal{O}_{R \rightarrow \infty}\left(R^{-(N+1) \alpha}\right) .
$$

Notation 3.14. In the situation of Proposition 3.13, we write

$$
U(X) \underset{R \rightarrow \infty}{\simeq} \sum_{p=1}^{P} A_{p} \mathfrak{s}^{p \alpha}(R, \theta)+\sum_{p=1}^{\infty} B_{p} \mathfrak{s}^{-p \alpha}(R, \theta) .
$$




\section{Multi-Scale ExPansion}

The multi-scale expansion in the domain $\mathscr{U}_{\varepsilon}$ is composed of two different types of terms: the slow terms involving the original variable $x$, and the profiles appearing in the rapid scaled variable $\frac{x}{\varepsilon}$. They are superposed via cut-off functions according to the Ansatz

$$
u_{\varepsilon}(x)=\chi\left(\frac{x}{\varepsilon}\right) \sum_{\ell=0}^{n} \varepsilon^{\ell \alpha} v^{\ell \alpha}(x)+\psi(x) \sum_{\ell=0}^{n} \varepsilon^{\ell \alpha} V^{\ell \alpha}\left(\frac{x}{\varepsilon}\right)+\mathcal{O}\left(\varepsilon^{n \alpha}\right),
$$

where the functions $\chi$ and $\psi$ are smooth and satisfy

$$
\left\{\begin{array}{ccc}
\chi(X)=1 \text { for }|X|>2 R^{*} & \text { and } & \chi(X)=0 \text { for }|X|<\frac{3 R^{*}}{2}, \\
\psi(x)=1 \text { for }|x|<\frac{r^{*}}{2} & \text { and } & \psi(x)=0 \text { for }|x|>r^{*}
\end{array}\right.
$$

The first sum in (4.1) has its support away from an $\varepsilon$-neighborhood of the limit point $\mathbf{0}$ and, conversely, the second brings a contribution in a neighborhood of $\mathbf{0}$ (independent of $\varepsilon)$. The transition region is the common support of the two sums which, thanks to (2.2), satisfies for any $\varepsilon \leqslant \varepsilon_{0} / 2$,

$$
\begin{aligned}
\mathscr{U}_{\varepsilon} \cap\left(\operatorname{supp} \chi\left(\frac{\dot{ }}{\varepsilon}\right) \cap \operatorname{supp} \psi\right) & \subset\left\{x \in \mathscr{U}_{\varepsilon}, \varepsilon R^{*} \leqslant|x| \leqslant r^{*}\right\} \\
& =\left\{x \in K, \varepsilon R^{*} \leqslant|x| \leqslant r^{*}\right\} .
\end{aligned}
$$

The construction principles of the terms is as follows: $v^{\ell \alpha}$ and $V^{\ell \alpha}$ are solutions of variational problems in slow variables $x \in \omega$ and fast variables $X \in \Omega$. The cut-off by $\chi\left(\frac{x}{\varepsilon}\right)=\chi(X)$ and $\psi(x)$ introduces an error in fast and slow variables. These errors can be corrected with the help of the expansions as $r \rightarrow 0$ of the terms $v^{\ell \alpha}$ and as $R \rightarrow \infty$ of the terms $V^{\ell \alpha}$. Both expansions in homogeneous terms do make sense in fast and slow variables simultaneously, which allows us to bridge the terms in the two sums in (4.1).

4.1. The construction of the first terms. Step 0. Let $v^{0}=u_{0}$ be the solution of the limit variational problem (2.5). Since $v^{0}$ is defined on the domain $\omega$, and not on $\mathscr{U}_{\varepsilon}$, we choose to consider the truncated function $\widetilde{v}^{0}=\chi\left(\frac{x}{\varepsilon}\right) v^{0}$ instead. We note that $\widetilde{v}^{0}$ satisfies the Dirichlet boundary condition $\widetilde{v}^{0}=0$ on $\partial \mathscr{U}_{\varepsilon}$ and belongs to $\mathrm{H}_{0}^{1}\left(\mathscr{U}_{\varepsilon}\right)$. We consider the first remainder $r_{\varepsilon}^{0}$ defined as

$$
u_{\varepsilon}(x)=\chi\left(\frac{x}{\varepsilon}\right) v^{0}(x)+r_{\varepsilon}^{0}(x)
$$

Thus the support of $\Delta r_{\varepsilon}^{0}$ is contained in the support of $\nabla \chi\left(\frac{x}{\varepsilon}\right)$. Using the commutator $[\Delta, \varphi]$ defined by $[\Delta, \varphi] f:=\Delta(\varphi f)-\varphi \Delta f$, we find

$$
-\Delta r_{\varepsilon}^{0}(x)=\left([\Delta, \chi(\dot{\bar{\varepsilon}})] v^{0}\right)(x) \quad=2 \nabla_{x} v^{0}(x) \cdot \nabla_{x}\left(\chi\left(\frac{x}{\varepsilon}\right)\right)+v^{0}(x) \Delta_{x}\left(\chi\left(\frac{x}{\varepsilon}\right)\right) .
$$

Since $f \equiv 0$ in a neighborhood of $\mathbf{0}$, according to Proposition 3.6 (and using Notation 3.7) there exists a sequence $\left(\mathbf{b}_{p}^{0}\right)_{p \geqslant 1}$ such that $v^{0}$ expands as $r \rightarrow 0$ as

$$
v^{0}(x) \underset{r \rightarrow 0}{\simeq} \sum_{p=1}^{\infty} \mathbf{b}_{p}^{0} \mathfrak{s}^{p \alpha}(x) .
$$


We insert the expansion (4.4) into (4.3). For each of its terms we use the fundamental relation which allows to convert the commutator in fast variables

$$
\left[\Delta, \chi\left(\frac{x}{\varepsilon}\right)\right] \mathfrak{s}^{p \alpha}(x)=\varepsilon^{-2} \varepsilon^{p \alpha}\left(\left[\Delta_{X}, \chi\right] \mathfrak{s}^{p \alpha}\right)\left(\frac{x}{\varepsilon}\right) .
$$

Thus the remainder (4.3) can be written as

$$
\Delta r_{\varepsilon}^{0}(x) \underset{r \rightarrow 0}{\simeq}-\varepsilon^{-2} \sum_{p=1}^{\infty} \varepsilon^{p \alpha} \mathbf{b}_{p}^{0}\left(\left[\Delta_{X}, \chi\right] \mathfrak{s}^{p \alpha}\right)\left(\frac{x}{\varepsilon}\right) .
$$

To complete step 0 , we set $V^{0}=0$ and we are going to consider the further terms for $p=1, \ldots$, as right hand sides of a problem on $\Omega$ in the fast variable $X=\frac{x}{\varepsilon}$.

Step 1. The first term in the remainder asymptotics (4.6) is

$$
\varepsilon^{-2} \varepsilon^{\alpha} \mathbf{b}_{1}^{0}\left(\left[\Delta_{X}, \chi\right] \mathfrak{s}^{\alpha}\right)(X) .
$$

This function is smooth with compact support. Let $V^{\alpha}$ be the solution of the variational problem in $\Omega, c f$. (3.4),

$$
\text { Find } V^{\alpha} \in V(\Omega) \text { such that }-\Delta_{X} V^{\alpha}=\mathbf{b}_{1}^{0}\left[\Delta_{X}, \chi\right] \mathfrak{s}^{\alpha} \text { in } \Omega \text {. }
$$

Then it is clear that $\Delta_{x}\left(\varepsilon^{\alpha} V^{\alpha}\left(\frac{x}{\varepsilon}\right)\right)$ coincides with the function (4.7). Therefore a better start for the asymptotic expansion of $u_{\varepsilon}$ reads

$$
\chi\left(\frac{x}{\varepsilon}\right) v^{0}(x)+\psi(x) \varepsilon^{\alpha} V^{\alpha}\left(\frac{x}{\varepsilon}\right),
$$

which satisfies the Dirichlet boundary conditions on $\partial \mathscr{U}_{\varepsilon}$, and the associated remainder $r_{\varepsilon}^{\alpha}$ is defined as

$$
u_{\varepsilon}(x)=\chi\left(\frac{x}{\varepsilon}\right) v^{0}(x)+\psi(x) \varepsilon^{\alpha} V^{\alpha}\left(\frac{x}{\varepsilon}\right)+r_{\varepsilon}^{\alpha}(x) .
$$

Since $\psi \equiv 1$ on the support of the right hand side (4.7), we find

$$
\Delta r_{\varepsilon}^{\alpha}(x)=-\left[\Delta, \chi\left(\frac{x}{\varepsilon}\right)\right]\left(v^{0}(x)-\mathbf{b}_{1}^{0} \mathfrak{s}^{\alpha}(x)\right)-[\Delta, \psi] \varepsilon^{\alpha} V^{\alpha}\left(\frac{x}{\varepsilon}\right) .
$$

Again, the commutator $\left[\Delta, \chi\left(\frac{x}{\varepsilon}\right)\right]\left(v^{0}(x)-\mathbf{b}_{1}^{0} \mathfrak{s}^{\alpha}(x)\right)$ will be converted in rapid variables, and since

$$
v^{0}(x)-\mathbf{b}_{1}^{0} \mathfrak{s}^{\alpha}(x) \underset{r \rightarrow 0}{\simeq} \sum_{p=2}^{\infty} \mathbf{b}_{p}^{0} \mathfrak{s}^{p \alpha}\left(\frac{x}{\varepsilon}\right),
$$

we have gained one power of $\varepsilon^{\alpha}$.

Next, we express the other part of the remainder (4.9) in slow variables. Thanks to Lemma 3.10, we have $V^{\alpha}(X)=\mathcal{O}_{R \rightarrow \infty}(1)$. Thus Proposition 3.13 yields that $V^{\alpha}$ expands at infinity as

$$
V^{\alpha}(X) \underset{R \rightarrow \infty}{\simeq} \sum_{p=1}^{\infty} \mathbf{B}_{p}^{1} \mathfrak{s}^{-p \alpha}(X)
$$

Since $\Delta \mathfrak{s}^{-p \alpha}=0$, we find

$$
[\Delta, \psi] \varepsilon^{\alpha} V^{\alpha}\left(\frac{x}{\varepsilon}\right) \underset{\varepsilon \rightarrow 0}{\simeq} \sum_{p=1}^{\infty} \varepsilon^{(1+p) \alpha} \mathbf{B}_{p}^{1}[\Delta, \psi] \mathfrak{s}^{-p \alpha}(x) .
$$


The terms in (4.12) start with $\varepsilon^{2 \alpha}$. They can be compensated by the solution of problems in $\omega$. We set $v^{\alpha}=0$.

Step 2. Next we define $v^{2 \alpha}$ as the solution of the problem in slow variables in $\omega$

$$
\text { Find } v^{2 \alpha} \in \mathrm{H}_{0}^{1}(\omega) \text { such that }-\Delta_{x} v^{2 \alpha}=\mathbf{B}_{1}^{1}[\Delta, \psi] \mathfrak{s}^{-\alpha},
$$

and $V^{2 \alpha}$ as the solution of the problem in fast variables in $\Omega$ (compare with (4.8))

$$
\text { Find } V^{2 \alpha} \in V(\Omega) \text { such that }-\Delta_{X} V^{2 \alpha}=\mathbf{b}_{2}^{0}[\Delta, \chi] \mathfrak{s}^{2 \alpha} \text {. }
$$

4.2. The general construction. The construction is done by induction. Let us assume the asymptotic expansion built up to order $n-1$, i.e.

$$
u_{\varepsilon}(x)=\chi\left(\frac{x}{\varepsilon}\right) \sum_{\ell=0}^{n-1} \varepsilon^{\ell \alpha} v^{\ell \alpha}(x)+\psi(x) \sum_{\ell=1}^{n-1} \varepsilon^{\ell \alpha} V^{\ell \alpha}\left(\frac{x}{\varepsilon}\right)+r_{\varepsilon}^{(n-1) \alpha}(x),
$$

with $v^{\ell \alpha} \in \mathrm{H}_{0}^{1}(\omega)$ and $V^{\ell \alpha} \in V(\Omega)$ whose Laplacians vanish in a neighborhood of zero and $\infty$, respectively. For $\ell=0, \ldots, n-1$, we expand the term $v^{\ell \alpha}$ into singular functions at the corner point (see Proposition 3.6)

$$
v^{\ell \alpha}(x) \underset{r \rightarrow 0}{\simeq} \sum_{p=1}^{+\infty} \mathbf{b}_{p}^{\ell} \mathfrak{s}^{p \alpha}(x),
$$

and, we also expand the profiles $V^{\ell \alpha}$ into dual singular functions at infinity (see Proposition 3.13)

$$
V^{\ell \alpha}(X) \underset{R \rightarrow+\infty}{\simeq} \sum_{p=1}^{+\infty} \mathbf{B}_{p}^{\ell} \mathfrak{s}^{-p \alpha}(X)
$$

The definitions for the next terms $v^{n \alpha}$ and $V^{n \alpha}$ generalize (4.13) and (4.14). The function $v^{n \alpha} \in \mathrm{H}_{0}^{1}(\omega)$ solves

$$
\Delta v^{n \alpha}(x)=-\Delta\left[\psi(x) \sum_{\ell=1}^{n-1} \mathbf{B}_{n-\ell^{\ell}}^{\mathfrak{s}^{-(n-\ell) \alpha}}(x)\right]
$$

and, $V^{n \alpha} \in V(\Omega)$ satisfies

$$
\Delta V^{n \alpha}(X)=-\Delta\left[\chi(X) \sum_{\ell=0}^{n-1} \mathbf{b}_{n-\ell^{\ell}}^{\mathfrak{s}^{(n-\ell) \alpha}}(X)\right] .
$$

Let us calculate the residual: The Laplacian of the remainder is given by

$$
\Delta r_{\varepsilon}^{(n-1) \alpha}(x)=\Delta u^{\varepsilon}-\sum_{\ell=0}^{n-1} \varepsilon^{\ell \alpha}\left[\Delta\left(\chi\left(\frac{x}{\varepsilon}\right) v^{\ell \alpha}(x)\right)+\Delta\left(\psi(x) V^{\ell \alpha}\left(\frac{x}{\varepsilon}\right)\right)\right] .
$$

Next, we expand this relation using (4.16), (4.17), and relations (4.18), (4.19) with $n$ replaced by $1,2, \ldots, n-1$. We obtain

$$
\Delta r_{\varepsilon}^{(n-1) \alpha}=-\sum_{\ell=0}^{n-1} \varepsilon^{\ell \alpha}\left[[\Delta, \chi(\dot{\bar{\varepsilon}})] v_{n-1-\ell}^{\ell \alpha}+[\Delta, \psi] V_{n-1-\ell}^{\ell \alpha}(\dot{\bar{\varepsilon}})\right],
$$


with

$$
\begin{aligned}
v_{k}^{\ell \alpha}(x) & :=v^{\ell \alpha}(x)-\sum_{p=1}^{k} \mathbf{b}_{p}^{\ell} \mathfrak{s}^{p \alpha}(x) \underset{r \rightarrow 0}{\simeq} \sum_{p=k+1}^{+\infty} \mathbf{b}_{p}^{\ell} \mathfrak{s}^{p \alpha}(x) \\
V_{k}^{\ell \alpha}(X) & :=V^{\ell \alpha}(X)-\sum_{p=1}^{k} \mathbf{B}_{p}^{\ell} \mathfrak{s}^{-p \alpha}(X) \underset{R \rightarrow \infty}{\simeq} \sum_{p=k+1}^{+\infty} \mathbf{B}_{p}^{\ell} \mathfrak{s}^{-p \alpha}(X) .
\end{aligned}
$$

The leading term of the remainder $\Delta r_{\varepsilon}^{(n-1) \alpha}$ corresponds to the lowest terms in the sums in the right hand sides of identities (4.22), and is therefore

$$
\Delta\left[\sum_{\ell=0}^{n-1} \varepsilon^{\ell \alpha} \mathbf{b}_{n-\ell}^{\ell} \mathfrak{s}^{(n-\ell) \alpha}(x) \chi\left(\frac{x}{\varepsilon}\right)\right]+\Delta\left[\sum_{\ell=1}^{n-1} \varepsilon^{\ell \alpha} \mathbf{B}_{n-\ell}^{\ell} \mathfrak{s}^{-(n-\ell) \alpha}\left(\frac{x}{\varepsilon}\right) \psi(x)\right]
$$

which leads after scaling to, compare with (4.18) and (4.19):

$$
\varepsilon^{n \alpha}\left(\Delta\left[\sum_{\ell=0}^{n-1} \mathbf{b}_{n-\ell}^{\ell} \mathfrak{s}^{(n-\ell) \alpha}\left(\frac{x}{\varepsilon}\right) \chi\left(\frac{x}{\varepsilon}\right)\right]+\Delta\left[\sum_{\ell=1}^{n-1} \mathbf{B}_{n-\ell}^{\ell} \mathfrak{s}^{-(n-\ell) \alpha}(x) \psi(x)\right]\right) .
$$

\subsection{Optimal error estimate.}

Theorem 4.1. The solution $u_{\varepsilon}$ of problem (2.3) admits the following multiscale expansion into powers of $\varepsilon$ (recall that $\pi / \alpha$ is the opening angle of $\omega$ at 0$)$ :

$$
u_{\varepsilon}(x)=\chi\left(\frac{x}{\varepsilon}\right) \sum_{\ell=0}^{n} \varepsilon^{\ell \alpha} v^{\ell \alpha}(x)+\psi(x) \sum_{\ell=0}^{n} \varepsilon^{\ell \alpha} V^{\ell \alpha}\left(\frac{x}{\varepsilon}\right)+r_{\varepsilon}^{n \alpha}(x),
$$

where the terms $v^{\ell \alpha}$ and $V^{\ell \alpha}$ do not depend on $\varepsilon$, and are defined in $\omega$ and $\Omega$ by Equations (4.18) and (4.19), respectively. Moreover, the remainder $r_{\varepsilon}^{n \alpha}$ satisfies the following estimate

$$
\left\|r_{\varepsilon}^{n \alpha}\right\|_{\mathrm{H}^{1}(\mathscr{U} \varepsilon)} \leqslant C \varepsilon^{(n+1) \alpha} .
$$

Proof. A basic technique to estimate the remainder consists in investigating the LaplaceDirichlet problem it solves. By construction, $r_{\varepsilon}^{n \alpha}$ satisfies the homogeneous Dirichlet condition and belongs to $\mathrm{H}_{0}^{1}\left(\mathscr{U}_{\varepsilon}\right)$. By uniform coercivity, there exists $C_{0}>0$ such that

$$
\left\|r_{\varepsilon}^{n \alpha}\right\|_{\mathrm{H}^{1}(\mathscr{U} \varepsilon)} \leqslant C_{0}\left\|\Delta r_{\varepsilon}^{n \alpha}\right\|_{\mathrm{H}^{-1}(\mathscr{U} \varepsilon)}, \quad \forall \varepsilon \leqslant \varepsilon_{0} .
$$

Since $\Delta r_{\varepsilon}^{n \alpha}$ has the expression (4.21) (with $n-1$ replaced by $n$ ) we have to estimate each of its terms in $\mathrm{H}^{-1}\left(\mathscr{U}_{\varepsilon}\right)$-norm.

- For all $v$, the commutator of $\Delta$ and $\chi(\dot{\bar{\varepsilon}})$ is given by

$$
([\Delta, \chi(\dot{\bar{\varepsilon}})] v)(x)=2 \varepsilon^{-1} \nabla v(x) \cdot(\nabla \chi)\left(\frac{x}{\varepsilon}\right)+\varepsilon^{-2} v(x)(\Delta \chi)\left(\frac{x}{\varepsilon}\right) .
$$

Hence, the support of $[\Delta, \chi(\dot{\bar{\varepsilon}})] v$ is included in the annulus $3 R^{*} \varepsilon / 2 \leqslant r \leqslant 2 R^{*} \varepsilon$. For $v_{k}^{\ell \alpha}$, which is a $\mathcal{O}_{r \rightarrow 0}\left(r^{(k+1) \alpha}\right)$, one obtains the $\mathrm{L}^{\infty}$-bound

$$
\left\|[\Delta, \chi(\dot{\bar{\varepsilon}})] v_{k}^{\ell \alpha}\right\|_{\mathrm{L}^{\infty}\left(\mathscr{U}_{\varepsilon}\right)} \leqslant C \varepsilon^{(k+1) \alpha-2} .
$$



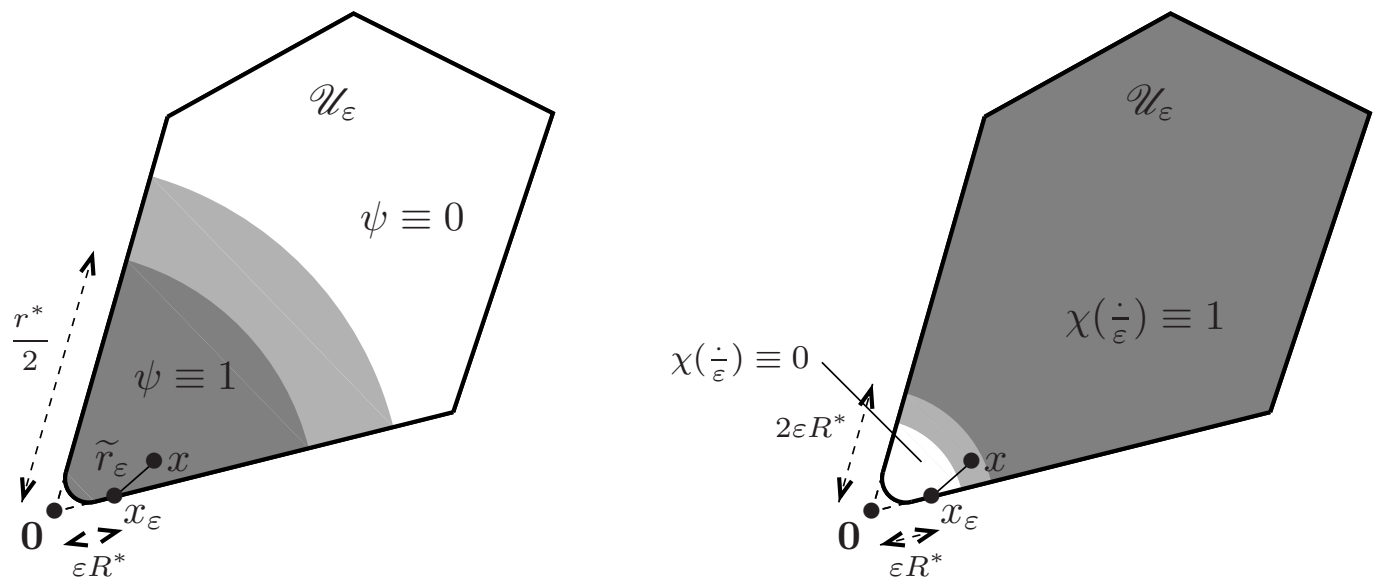

FIGURE 3. Point $x_{\varepsilon}$, distance $\widetilde{r}_{\varepsilon}$, and supports of cut-off functions $\psi$ and $\chi(\dot{\bar{\varepsilon}})$.

Let us choose $X_{0}$ such that $X_{0} \in \partial \Omega$ and $\left|X_{0}\right|=R^{*}$ (such a point does exist since $\Omega$ coincides with $K$ in the region $R>R^{*}$ ). Then the point $x_{\varepsilon}=X_{0} / \varepsilon$ belongs to $\partial \mathscr{U}_{\varepsilon}$, see Figure 3. Moreover, if we set

$$
\widetilde{r}_{\varepsilon}(x)=\left|x-x_{\varepsilon}\right|
$$

we find that $\widetilde{r}_{\varepsilon}$ is equivalent to $r$ in the support of $[\Delta, \chi(\dot{\varepsilon})]$, uniformly in $\varepsilon$. Since $x_{\varepsilon} \in \partial \mathscr{U}_{\varepsilon}$, there holds

$$
\left\|\frac{w}{\widetilde{r}_{\varepsilon}}\right\|_{\mathrm{L}^{2}\left(\mathscr{U}_{\varepsilon}\right)} \leqslant C_{1}\|w\|_{\mathrm{H}^{1}\left(\mathscr{U}_{\varepsilon}\right)}, \quad \forall w \in \mathrm{H}_{0}^{1}\left(\mathscr{U}_{\varepsilon}\right),
$$

with a constant $C_{1}$ independent of $\varepsilon<\varepsilon_{0} / 2$ and $w$. Let $w \in \mathrm{H}_{0}^{1}\left(\mathscr{U}_{\varepsilon}\right)$. We deduce via Hölder inequality

$$
\begin{aligned}
\left\langle[\Delta, \chi(\dot{\bar{\varepsilon}})] v_{k}^{\ell \alpha}, w\right\rangle & =\left\langle\widetilde{r}_{\varepsilon}[\Delta, \chi(\dot{\bar{\varepsilon}})] v_{k}^{\ell \alpha}, \frac{w}{\widetilde{r}_{\varepsilon}}\right\rangle \\
& \leqslant C\left\|[\Delta, \chi(\dot{\bar{\varepsilon}})] v_{k}^{\ell \alpha}\right\|_{\mathrm{L}^{\infty}(\mathscr{U} \varepsilon)}\left\|\widetilde{r}_{\varepsilon}\right\|_{\mathrm{L}^{2}(\mathscr{U} \varepsilon)}\|w\|_{\mathrm{H}^{1}(\mathscr{U} \varepsilon)} \\
& \leqslant C \varepsilon^{(k+1) \alpha-2} \varepsilon^{2}\|w\|_{\mathrm{H}^{1}\left(\mathscr{U}_{\varepsilon}\right)^{*}}
\end{aligned}
$$

Hence

$$
\left\|[\Delta, \chi(\dot{\bar{\varepsilon}})] v_{k}^{\ell \alpha}\right\|_{\mathrm{H}^{-1}\left(\mathscr{U}_{\varepsilon}\right)} \leqslant C \varepsilon^{(k+1) \alpha} .
$$

- Using that the function $V_{k}^{\ell \alpha}$ is a $\mathcal{O}_{R \rightarrow \infty}\left(R^{(k+1) \alpha}\right)$, we easily deduce the estimate

$$
\left\|[\Delta, \psi] V_{k}^{\ell \alpha}(\dot{\bar{\varepsilon}})\right\|_{\mathrm{L}^{2}(\mathscr{U} \varepsilon)} \leqslant C \varepsilon^{(k+1) \alpha} .
$$

Hence

$$
\left\|[\Delta, \psi] V_{k}^{\ell \alpha}(\dot{\bar{\varepsilon}})\right\|_{\mathrm{H}^{-1}(\mathscr{U} \varepsilon)} \leqslant C \varepsilon^{(k+1) \alpha} .
$$

One deduces immediately from (4.21), (4.28) and (4.29)

$$
\left\|\Delta r_{\varepsilon}^{n \alpha}\right\|_{\mathrm{H}^{-1}(\mathscr{U} \varepsilon)} \leqslant C \varepsilon^{(k+1) \alpha}
$$


and using the a priori estimate (4.25), we obtain the bound (4.24), which ends the proof.

\section{MATCHING OF ASYMPTOTIC EXPANSIONS}

5.1. Formal derivation of the asymptotic expansions. We will represent the solution $u_{\varepsilon}$ as a formal series in each zone of interest, that is the corner expansion (or inner expansion) near the origin $\mathbf{0}$ and the outer expansion away from $\mathbf{0}$. We write these two formal series in the form

$$
u_{\varepsilon}(x) \simeq \sum_{\ell=-\infty}^{+\infty} \varepsilon^{\ell \alpha} U^{\ell \alpha}\left(\frac{x}{\varepsilon}\right) \quad \text { and } \quad u_{\varepsilon}(x) \simeq \sum_{\ell=-\infty}^{+\infty} \varepsilon^{\ell \alpha} u^{\ell \alpha}(x)
$$

This Ansatz is suggested by the homogeneity of the singular functions, see (2.7). We will give a sense to the infinite sums in terms of asymptotic expansions later on.

Since the $\mathrm{H}^{1}$-norm of $u_{\varepsilon}$ is uniformly bounded with respect to $\varepsilon$, we know that all the $u^{\ell \alpha}$ and $U^{\ell \alpha}$ for $\ell<0$ are just zero. Moreover, it is clear that the terms of the asymptotic expansions must satisfy

$$
\left\{\begin{array}{l}
-\Delta u^{0}=f \text { in } \omega \text { and } u^{0}=0 \text { on } \partial \omega \\
\forall \ell>0, \Delta u^{\ell \alpha}=0 \text { in } \omega \text { and } u^{\ell \alpha}=0 \text { on } \partial \omega \backslash\{0\} \\
\forall \ell \geqslant 0, \Delta U^{\ell \alpha}=0 \text { in } \Omega \text { and } U^{\ell \alpha}=0 \text { on } \partial \Omega
\end{array}\right.
$$

Now we need to ensure the matching of the two formal series in the transition zone

$$
\varepsilon \ll r \ll 1 .
$$

To do so, we expand the terms $u^{\ell \alpha}$ and $U^{\ell \alpha}$. Thanks to Propositions 3.6 and 3.13 - note that $r \ll 1$ and $\frac{r}{\varepsilon} \gg 1-$ these expansions read $^{2}$

$$
\left\{\begin{array}{l}
u^{\ell \alpha}(x)=\sum_{p=1}^{+\infty}\left(a_{p}^{\ell} \mathfrak{s}^{-p \alpha}(r, \theta)+b_{p}^{\ell} \mathfrak{s}^{p \alpha}(r, \theta)\right), \\
U^{\ell \alpha}(X)=\sum_{p=1}^{+\infty}\left(A_{p}^{\ell} \mathfrak{s}^{p \alpha}(R, \theta)+B_{p}^{\ell} \mathfrak{s}^{-p \alpha}(R, \theta)\right) .
\end{array}\right.
$$

\footnotetext{
${ }^{2}$ Note that we do not use the boldface notation for the coefficients $b_{p}^{0}$, because we do not yet know whether they coincide with the coefficients $\mathbf{b}_{p}^{0}$ already defined in Section 4. The coincidence will be shown in Section 6.
} 
We use the homogeneity of the functions $\mathfrak{s}^{p \alpha}$ and transform the rapid variable $\frac{r}{\varepsilon}$ into the slow one $r$. Ensuring the equality of the two formal series (5.1), we get

$$
\left\{\begin{aligned}
\sum_{\ell=-\infty}^{+\infty} & \left(\varepsilon^{\ell \alpha} \sum_{p=1}^{+\infty}\left(a_{p^{\ell}} \mathfrak{s}^{-p \alpha}(r, \theta)+b_{p}^{\ell} \mathfrak{s}^{p \alpha}(r, \theta)\right)\right) \\
& =\sum_{\ell=-\infty}^{+\infty}\left(\varepsilon^{\ell \alpha} \sum_{p=1}^{+\infty}\left(A_{p}^{\ell} \mathfrak{s}^{p \alpha}\left(\frac{r}{\varepsilon}, \theta\right)+B_{p}^{\ell} \mathfrak{s}^{-p \alpha}\left(\frac{r}{\varepsilon}, \theta\right)\right)\right) \\
& =\sum_{\ell=-\infty}^{+\infty}\left(\varepsilon^{\ell \alpha} \sum_{p=1}^{+\infty}\left(\varepsilon^{-p \alpha} A_{p}^{\ell} \mathfrak{s}^{p \alpha}(r, \theta)+\varepsilon^{p \alpha} B_{p}^{\ell} \mathfrak{s}^{-p \alpha}(r, \theta)\right)\right) \\
& =\sum_{\ell=-\infty}^{+\infty}\left(\varepsilon^{\ell \alpha} \sum_{p=1}^{+\infty}\left(A_{p}^{\ell+p} \mathfrak{s}^{p \alpha}(r, \theta)+B_{p}^{\ell-p} \mathfrak{s}^{-p \alpha}(r, \theta)\right)\right) .
\end{aligned}\right.
$$

Identifying the terms of the two series leads to

$$
b_{p}^{\ell}=A_{p}^{\ell+p} \quad \text { and } \quad a_{p}^{\ell}=B_{p}^{\ell-p},
$$

i. e.

$$
\left\{\begin{array}{c}
a_{p}^{\ell}=B_{p}^{\ell-p} \text { if } p \leqslant \ell \text { and } a_{p}^{\ell}=0 \text { if } p>\ell \\
A_{p}^{\ell}=b_{p}^{\ell-p} \text { if } p \leqslant \ell \text { and } A_{p}^{\ell}=0 \text { if } p>\ell
\end{array}\right.
$$

knowing that $b_{p}^{\ell-p}=B_{p}^{\ell-p}=0$ for $p>\ell$, since the terms $u^{n \alpha}$ and $U^{n \alpha}$ are 0 for $n<0$.

Remark 5.1. Here, we have chosen to derive the matching relations without any knowledge of the matched asymptotic technique. However, one can derive the relations (5.7) using the Van Dyke principle, see [25].

5.2. Definition of the asymptotic terms. For $\ell \in \mathbb{N}$, the functions $u^{\ell \alpha}$ and $U^{\ell \alpha}$ are defined inductively. The following algorithm defines step by step $u^{\ell \alpha}: \omega \rightarrow \mathbb{R}, U^{\ell \alpha}: \Omega \rightarrow \mathbb{R}$, $b^{\ell}=\left(b_{p}^{\ell}\right)_{p \in \mathbb{N}^{*}}$, and $B^{\ell}=\left(B_{p}^{\ell}\right)_{p \in \mathbb{N}^{*}}$ for $\ell \in \mathbb{N}$.

Step 0. $u^{0} \in V_{\text {loc }, 0}(\omega)$ is defined via Proposition 3.4 (in the particular case of Remark 3.5) as the unique function satisfying

$$
\Delta u^{0}=-f \text { in } \omega \text { and } u^{0}=\mathcal{O}_{r \rightarrow 0}(1) .
$$

Moreover, $U^{0}$ is chosen to be 0 . Let $b^{0}$ be the sequence of numbers defined by Proposition 3.6 and $B^{0}$ be zero:

$$
b^{0}=\left(b_{p}^{0}\right)_{p \in \mathbb{N}^{*}} \quad \text { and } \quad B^{0}=\left(B_{p}^{0}\right)_{p \in \mathbb{N}^{*}}=0 .
$$

Step $\ell$. We denote by $a^{\ell}=\left(a_{p}^{\ell}\right)_{p \in \mathbb{N}^{*}}$ and $A^{\ell}=\left(A_{p}^{\ell}\right)_{p \in \mathbb{N}^{*}}$ the two finite sequences of real numbers such that

$$
\left\{\begin{array}{l}
a_{p}^{\ell}=B_{p}^{\ell-p} \text { if } 1 \leqslant p \leqslant \ell-1 \quad \text { and } \quad a_{p}^{\ell}=0 \text { if } p \geqslant \ell \\
A_{p}^{\ell}=b_{p}^{\ell-p} \text { if } 1 \leqslant p \leqslant \ell \quad \text { and } \quad A_{p}^{\ell}=0 \text { if } p \geqslant \ell+1
\end{array}\right.
$$


The functions $u^{\ell \alpha}$ and $U^{\ell \alpha}$ are defined via Propositions 3.4 and 3.11 as the unique solutions of the problems

$$
\left\{\begin{array}{l}
\text { Find } u^{\ell \alpha} \in V_{\mathrm{loc}, 0}(\omega) \text { such that } \\
\Delta u^{\ell \alpha}=0 \text { in } \omega \text { and } u^{\ell \alpha}-\sum_{p=1}^{\ell-1} a_{p}^{\ell} \mathfrak{s}^{-p \alpha}=\mathcal{O}_{r \rightarrow 0}(1),
\end{array}\right.
$$

and

$$
\left\{\begin{array}{l}
\text { Find } U^{\ell \alpha} \in V_{\mathrm{loc}, \infty}(\Omega) \text { such that } \\
\Delta U^{\ell \alpha}=0 \text { in } \Omega \text { and } U^{\ell \alpha}-\sum_{p=1}^{\ell} A_{p}^{\ell} \mathfrak{s}^{p \alpha}=\mathcal{O}_{R \rightarrow \infty}(1) .
\end{array}\right.
$$

Finally, we define the sequences $b^{\ell}$ and $B^{\ell}$ associated with $u^{\ell \alpha}$ and $U^{\ell \alpha}$ in Propositions 3.6 and 3.13

$$
b^{\ell}=\left(b_{p}^{\ell}\right)_{p \in \mathbb{N}^{*}} \quad \text { and } \quad B^{\ell}=\left(B_{p}^{\ell}\right)_{p \in \mathbb{N}^{*}}
$$

5.3. Global error estimates. The main idea to prove error estimates is to define a global approximation $\widehat{u}_{n \alpha}^{\varepsilon} \in \mathrm{H}_{0}^{1}\left(\mathscr{U}_{\varepsilon}\right)$ of $u_{\varepsilon}$ by the formula

$$
\widehat{u}_{n \alpha}^{\varepsilon}(x)=\varphi\left(\frac{r}{\eta(\varepsilon)}\right) \sum_{\ell=0}^{n} \varepsilon^{\ell \alpha} u^{\ell \alpha}(x)+\left(1-\varphi\left(\frac{r}{\eta(\varepsilon)}\right)\right) \sum_{\ell=1}^{n} \varepsilon^{\ell \alpha} U^{\ell \alpha}\left(\frac{x}{\varepsilon}\right),
$$

where $\varphi$ is a smooth cut-off function with $\varphi(\rho)=0$ for $\rho<1$ and $\varphi(\rho)=1$ for $\rho>2$ and $\eta$ is a smooth function of $\varepsilon$ such that

$$
\lim _{\varepsilon \rightarrow 0} \eta(\varepsilon)=0 \quad \text { and } \quad \lim _{\varepsilon \rightarrow 0} \frac{\eta(\varepsilon)}{\varepsilon}=+\infty .
$$

Theorem 5.2. There exists a constant $C$ such that

$$
\left\|u_{\varepsilon}-\widehat{u}_{n \alpha}^{\varepsilon}\right\|_{\mathrm{H}^{1}(\mathscr{U} \varepsilon)} \leqslant C\left[(\eta(\varepsilon))^{(n+1) \alpha}+\left(\frac{\varepsilon}{\eta(\varepsilon)}\right)^{(n+1) \alpha}\right] .
$$

Remark 5.3. One can optimize the estimate (5.16) by choosing the best $\eta$ : For $\eta(\varepsilon)=\varepsilon^{1 / 2}$, there exists a constant $C$ such that

$$
\left\|u_{\varepsilon}-\widehat{u}_{n \alpha}^{\varepsilon}\right\|_{H^{1}(\mathscr{U} \varepsilon)} \leqslant C \varepsilon^{(n+1) \alpha / 2} .
$$

Proof. First, we denote by $\widehat{e}_{n \alpha}^{\varepsilon}$ the approximation error at step $n$

$$
\widehat{e}_{n \alpha}^{\varepsilon}(x)=\widehat{u}_{n \alpha}^{\varepsilon}(x)-u_{\varepsilon}(x)
$$

and by $\mathcal{E}_{n \alpha}^{\varepsilon}$ the corresponding matching error

$$
\mathcal{E}_{\alpha n}^{\varepsilon}(x)=\sum_{\ell=0}^{n} \varepsilon^{\ell \alpha}\left[u^{\ell \alpha}(x)-U^{\ell \alpha}\left(\frac{x}{\varepsilon}\right)\right] .
$$

Of course, the matching error makes sense and is small only in the intermediate region; we shall express the $\mathrm{H}^{1}$-norm of $\widehat{e}_{n \alpha}^{\varepsilon}$ over $\mathscr{U}_{\varepsilon}$ in terms of $\mathcal{E}_{n \alpha}^{\varepsilon}$ in this region. By harmonicity of 
$u_{\varepsilon}, u^{\ell \alpha}$, and $U^{\ell \alpha}$, we obtain

$$
\Delta \widehat{e}_{n \alpha}^{\varepsilon}(x)=\frac{2}{\eta(\varepsilon)}[\nabla \varphi]\left(\frac{r}{\eta(\varepsilon)}\right) \nabla \mathcal{E}_{n \alpha}^{\varepsilon}(x)+\frac{1}{[\eta(\varepsilon)]^{2}}[\Delta \varphi]\left(\frac{r}{\eta(\varepsilon)}\right) \mathcal{E}_{n \alpha}^{\varepsilon}(x) .
$$

Since $\widehat{e}_{n \alpha}^{\varepsilon}$ belongs to $\mathrm{H}_{0}^{1}\left(\mathscr{U}_{\varepsilon}\right)$, the Green formula leads to

$$
\left\{\begin{aligned}
\int_{\mathscr{U}_{\varepsilon}}\left(\nabla \widehat{e}_{n \alpha}^{\varepsilon}\right)^{2} d x & =\frac{2}{\eta(\varepsilon)} \int_{\mathscr{U}_{\varepsilon}}[\nabla \varphi]\left(\frac{r}{\eta(\varepsilon)}\right) \nabla \mathcal{E}_{n \alpha}^{\varepsilon} \widehat{e}_{n \alpha}^{\varepsilon} \\
& +\frac{1}{[\eta(\varepsilon)]^{2}} \int_{\mathscr{U}_{\varepsilon}}[\Delta \varphi]\left(\frac{r}{\eta(\varepsilon)}\right) \mathcal{E}_{n \alpha}^{\varepsilon} \widehat{e}_{n \alpha}^{\varepsilon} d x \\
& \leqslant \frac{C}{[\eta(\varepsilon)]^{2}}\left[\left\|\mathcal{E}_{n \alpha}^{\varepsilon}\right\|_{\infty, \eta(\varepsilon)}+\eta(\varepsilon)\left\|\nabla \mathcal{E}_{n \alpha}^{\varepsilon}\right\|_{\infty, \eta(\varepsilon)}\right]\left\|\widehat{e}_{n \alpha}^{\varepsilon}\right\|_{1, \eta(\varepsilon)},
\end{aligned}\right.
$$

with the notation, for $p \in[1, \infty]$

$$
\|u\|_{p, \eta(\varepsilon)}=\|u\|_{\mathrm{L}^{p}(\{x \in \omega ; \eta(\varepsilon) \leqslant r \leqslant 2 \eta(\varepsilon)\})} .
$$

Using a Poincaré inequality on $\mathscr{U}_{\varepsilon}$ (uniform with respect to $\varepsilon$ ), we get

$$
\left\|\widehat{e}_{n \alpha}^{\varepsilon}\right\|_{\mathrm{H}^{1}(\mathscr{U} \varepsilon)}^{2} \leqslant \frac{C}{(\eta(\varepsilon))^{2}}\left[\left\|\mathcal{E}_{n \alpha}^{\varepsilon}\right\|_{\infty, \eta(\varepsilon)}+\eta(\varepsilon)\left\|\nabla \mathcal{E}_{n \alpha}^{\varepsilon}\right\|_{\infty, \eta(\varepsilon)}\right] \times\left\|\widehat{e}_{n \alpha}^{\varepsilon}\right\|_{1, \eta(\varepsilon)} .
$$

The conclusion follows from the following two lemmas (proved below).

Lemma 5.4. There exists a constant $C$ such that for all $u \in \mathrm{H}_{0}^{1}\left(\mathscr{U}_{\varepsilon}\right)$, the norm $\|u\|_{1, \eta(\varepsilon)}$, defined in (5.18), can be estimated as follows

$$
\|u\|_{1, \eta(\varepsilon)} \leqslant C[\eta(\varepsilon)]^{2}\|u\|_{\mathrm{H}^{1}(\mathscr{U} \varepsilon)} .
$$

Lemma 5.5. There exists a constant $C$ such that-for the definition of the norms, see (5.18),

$$
\begin{gathered}
\left\|\mathcal{E}_{n \alpha}^{\varepsilon}\right\|_{\infty, \eta(\varepsilon)} \leqslant C\left[(\eta(\varepsilon))^{(n+1) \alpha}+\left(\frac{\varepsilon}{\eta(\varepsilon)}\right)^{(n+1) \alpha}\right], \\
\left\|\nabla \mathcal{E}_{n \alpha}^{\varepsilon}\right\|_{\infty, \eta(\varepsilon)} \leqslant C \frac{1}{\eta(\varepsilon)}\left[(\eta(\varepsilon))^{(n+1) \alpha}+\left(\frac{\varepsilon}{\eta(\varepsilon)}\right)^{(n+1) \alpha}\right] .
\end{gathered}
$$

Proof. [lemma 5.4] For all $u \in \mathrm{H}_{0}^{1}\left(\mathscr{U}_{\varepsilon}\right)$ and for all $r \in[\eta(\varepsilon), 2 \eta(\varepsilon)]$

$$
\int_{0}^{\frac{\pi}{\alpha}}|u(r, \theta)| d \theta \leqslant \int_{0}^{\frac{\pi}{\alpha}}\left[\int_{0}^{\theta}\left|\frac{\partial u}{\partial \theta}\left(r, \theta^{\prime}\right)\right| d \theta^{\prime}\right] d \theta \leqslant \frac{\pi}{\alpha} \int_{0}^{\frac{\pi}{\alpha}}\left|\frac{\partial u}{\partial \theta}(r, \theta)\right| d \theta .
$$

Hence, we have

$$
\begin{array}{rl}
\int_{\eta(\varepsilon)}^{2 \eta(\varepsilon)} \int_{0}^{\frac{\pi}{\alpha}}|u(r, \theta)| r d & d \theta \leqslant \frac{\pi}{\alpha} \int_{\eta(\varepsilon)}^{2 \eta(\varepsilon)} \int_{0}^{\frac{\pi}{\alpha}}\left|\frac{\partial u}{\partial \theta}(r, \theta)\right| r d r d \theta \\
\leqslant \frac{\pi}{\alpha} \int_{\eta(\varepsilon)}^{2 \eta(\varepsilon)} \int_{0}^{\frac{\pi}{\alpha}} \frac{2 \eta(\varepsilon)}{r}\left|\frac{\partial u}{\partial \theta}(r, \theta)\right| r d r d \theta \leqslant C \eta(\varepsilon)\|\nabla u\|_{1, \eta(\varepsilon) .}
\end{array}
$$

We conclude using the Cauchy-Schwarz inequality that

$$
\|u\|_{1, \eta(\varepsilon)} \leqslant C \eta(\varepsilon)\|\nabla u\|_{1, \eta(\varepsilon)} \leqslant C[\eta(\varepsilon)]^{2}\|\nabla u\|_{2, \eta(\varepsilon)} .
$$


Proof. [lemma 5.5] We will give the proof of (5.20). Inequality (5.21) can be obtained using the same technique. The first step is to expand the $u^{\ell \alpha}$ and $U^{\ell \alpha}$ using (3.12) and (3.19). By definition of $u^{\ell \alpha}$ and $U^{\ell \alpha}$ - see (5.11) and (5.12), and taking (5.10) into account one finds

$$
\begin{aligned}
& u^{\ell \alpha}(x)=\sum_{p=1}^{\ell} B_{p}^{\ell-p} \mathfrak{s}^{-p \alpha}(r, \theta)+\sum_{p=1}^{n-\ell} b_{p}^{\ell} \mathfrak{s}^{p \alpha}(r, \theta)+\mathcal{O}_{r \rightarrow 0}\left(r^{(n+1-\ell) \alpha}\right), \\
& U^{\ell \alpha}(X)=\sum_{p=1}^{\ell} b_{p}^{\ell-p} \mathfrak{s}^{p \alpha}(R, \theta)+\sum_{p=1}^{n-\ell} B_{p}^{\ell} \mathfrak{s}^{-p \alpha}(R, \theta)+\mathcal{O}_{R \rightarrow \infty}\left(R^{(\ell-n-1) \alpha}\right) .
\end{aligned}
$$

Since $\eta(\varepsilon)$ tends to 0 and $\eta(\varepsilon) / \varepsilon$ tends to $+\infty$ when $\varepsilon$ tends to 0 , one has for $\eta(\varepsilon) \leqslant r \leqslant$ $2 \eta(\varepsilon)$

$$
\left\{\begin{array}{l}
\left|u^{\ell \alpha}(x)-\sum_{p=1}^{\ell} B_{p}^{\ell-p} \mathfrak{s}^{-p \alpha}(r, \theta)-\sum_{p=1}^{n-\ell} b_{p}^{\ell} \mathfrak{s}^{p \alpha}(r, \theta)\right| \leqslant C[\eta(\varepsilon)]^{(n+1-\ell) \alpha} \\
\left|U^{\ell \alpha}\left(\frac{x}{\varepsilon}\right)-\sum_{p=1}^{\ell} b_{p}^{\ell-p} \mathfrak{s}^{p \alpha}\left(\frac{r}{\varepsilon}, \theta\right)-\sum_{p=1}^{n-\ell} B_{p}^{\ell} \mathfrak{s}^{-p \alpha}\left(\frac{r}{\varepsilon}, \theta\right)\right| \leqslant C\left[\frac{\varepsilon}{\eta(\varepsilon)}\right]^{(n+1-\ell) \alpha}
\end{array}\right.
$$

Let $S$ be given by

$$
\begin{aligned}
S= & \sum_{\ell=0}^{n} \varepsilon^{\ell \alpha}\left(\sum_{p=1}^{\ell} B_{p}^{\ell-p} \mathfrak{s}^{-p \alpha}(r, \theta)+\sum_{p=1}^{n-\ell} b_{p}^{\ell} \mathfrak{s}^{p \alpha}(r, \theta)\right) \\
& -\sum_{\ell=0}^{n} \varepsilon^{\ell \alpha}\left(\sum_{p=1}^{\ell} b_{p}^{\ell-p} \mathfrak{s}^{p \alpha}\left(\frac{r}{\varepsilon}, \theta\right)+\sum_{p=1}^{n-\ell} B_{p}^{\ell} \mathfrak{s}^{-p \alpha}\left(\frac{r}{\varepsilon}, \theta\right)\right) .
\end{aligned}
$$

From (5.22) and triangle inequalities, we obtain

$$
\begin{gathered}
\left\|\mathcal{E}_{n \alpha}^{\varepsilon}(r, \theta)-S\right\|_{\infty, \eta(\varepsilon)} \leqslant C\left\{\sum_{\ell=0}^{n} \varepsilon^{\ell \alpha}[\eta(\varepsilon)]^{(n+1-\ell) \alpha}+\sum_{\ell=0}^{n} \varepsilon^{\ell \alpha}\left[\frac{\varepsilon}{\eta(\varepsilon)}\right]^{(n+1-\ell) \alpha}\right\} \\
\leqslant C\left\{\sum_{\ell=0}^{n}\left[\frac{\varepsilon}{\eta(\varepsilon)}\right]^{\ell \alpha}[\eta(\varepsilon)]^{(n+1) \alpha}+\sum_{\ell=0}^{n} \eta(\varepsilon)^{\ell \alpha}\left[\frac{\varepsilon}{\eta(\varepsilon)}\right]^{(n+1) \alpha}\right\} \\
\leqslant C\left\{[\eta(\varepsilon)]^{(n+1) \alpha}+\left[\frac{\varepsilon}{\eta(\varepsilon)}\right]^{(n+1) \alpha}\right\} .
\end{gathered}
$$

Now it remains to show that $S=0$. By definition - see $(2.7)-$ the singular functions $\mathfrak{s}^{ \pm p \alpha}$ satisfy the homogeneity property

$$
\mathfrak{s}^{-p \alpha}\left(\frac{r}{\varepsilon}, \theta\right)=\varepsilon^{p \alpha} \mathfrak{s}^{-p \alpha}(r, \theta) \quad \text { and } \quad \mathfrak{s}^{p \alpha}(r, \theta)=\varepsilon^{p \alpha} \mathfrak{s}^{p \alpha}\left(\frac{r}{\varepsilon}, \theta\right)
$$


Therefore, $S$ is given by

$$
\begin{aligned}
S= & \sum_{\ell=0}^{n} \sum_{p=1}^{\ell} \varepsilon^{(\ell-p) \alpha} B_{p}^{\ell-p} \mathfrak{s}^{-p \alpha}\left(\frac{r}{\varepsilon}, \theta\right)+\sum_{\ell=0}^{n} \sum_{p=1}^{n} \varepsilon^{\ell \alpha} b_{p}^{\ell} \mathfrak{s}^{p \alpha}(r, \theta) \\
& -\sum_{\ell=0}^{n} \sum_{p=1}^{\ell} \varepsilon^{(\ell-p) \alpha} b_{p}^{\ell-p} \mathfrak{s}^{p \alpha}(r, \theta)-\sum_{\ell=0}^{n} \sum_{p=1}^{n} \varepsilon^{\ell \alpha} B_{p}^{\ell} \mathfrak{s}^{-p \alpha}\left(\frac{r}{\varepsilon}, \theta\right) .
\end{aligned}
$$

The change of variables $\ell-p \mapsto \ell$ in the first and third terms leads to $S=0$.

5.4. Local error estimates. In this paragraph $\mathscr{B}_{r}$ will denote the ball of radius $r$ and of center $O$. Starting from the global error estimates obtained in (5.17), it is easy to get estimates far from the corner and near the corner

Theorem 5.6. For any $r_{0}>0$, there exists $C>0$ such that

$$
\left\|u_{\varepsilon}(r, \theta)-\sum_{\ell=0}^{n} \varepsilon^{\ell \alpha} u^{\ell \alpha}(r, \theta)\right\|_{\mathrm{H}^{1}\left(\omega \backslash \mathscr{B}_{r_{0}}\right)}=\mathcal{O}\left(\varepsilon^{(n+1) \alpha}\right) .
$$

For any $R_{0}>0$, there exists $C>0$ such that

$$
\left\|u_{\varepsilon}(\varepsilon R, \theta)-\sum_{\ell=0}^{n} \varepsilon^{\ell \alpha} U^{\ell \alpha}(R, \theta)\right\|_{\mathrm{H}^{1}\left(\Omega \cap \mathscr{B}_{R_{0}}\right)}=\mathcal{O}\left(\varepsilon^{(n+1) \alpha}\right) .
$$

Proof. To prove (5.25), we remark that, for $\varepsilon$ small enough, the only contribution comes from the terms $u^{\ell \alpha}$

$$
\widehat{u}_{n \alpha}^{\varepsilon}=\sum_{\ell=1}^{n} \varepsilon^{\ell \alpha} u^{\ell \alpha} \quad \text { in } \quad \mathscr{U}_{\varepsilon} \backslash \mathscr{B}_{r_{0}}=\omega \backslash \mathscr{B}_{r_{0}} .
$$

Consequently,

$$
\begin{aligned}
\| u_{\varepsilon}- & \widehat{u}_{n \alpha}^{\varepsilon} \|_{\mathrm{H}^{1}\left(\omega \backslash \mathscr{B}_{r_{0}}\right)} \\
& \leqslant\left\|u_{\varepsilon}-\widehat{u}_{(2 n+2) \alpha}^{\varepsilon}\right\|_{\mathrm{H}^{1}\left(\omega \backslash \mathscr{B}_{r_{0}}\right)}+\left\|\widehat{u}_{(2 n+2) \alpha}^{\varepsilon}-\widehat{u}_{n \alpha}^{\varepsilon}\right\|_{\mathrm{H}^{1}\left(\omega \backslash \mathscr{B}_{r_{0}}\right)} \\
& \leqslant\left\|u_{\varepsilon}-\widehat{u}_{(2 n+2) \alpha}^{\varepsilon}\right\|_{\mathrm{H}^{1}\left(\mathscr{U}_{\varepsilon}\right)}+\left\|\widehat{u}_{(2 n+2) \alpha}^{\varepsilon}-\widehat{u}_{n \alpha}^{\varepsilon}\right\|_{\mathrm{H}^{1}\left(\omega \backslash \mathscr{B}_{r_{0}}\right)} .
\end{aligned}
$$

On the other hand, it follows from (5.27)

$$
\widehat{u}_{(2 n+2) \alpha}^{\varepsilon}-\widehat{u}_{n \alpha}^{\varepsilon}=\sum_{\ell=n+1}^{2 n+2} \varepsilon^{\ell \alpha} u^{\ell \alpha} \quad \text { in } \quad \omega \backslash \mathscr{B}_{r_{0}},
$$

and, since the $u^{\ell \alpha}$ 's do not depend on $\varepsilon$

$$
\left\|\widehat{u}_{(2 n+2) \alpha}^{\varepsilon}-\widehat{u}_{n \alpha}^{\varepsilon}\right\|_{\mathrm{H}^{1}\left(\omega \backslash \mathscr{B}_{r_{0}}\right)} \leqslant C \varepsilon^{(n+1) \alpha} .
$$

Due to (5.17), one finally has

$$
\left\|u_{\varepsilon}-\widehat{u}_{(2 n+2) \alpha}^{\varepsilon}\right\|_{\mathrm{H}^{1}\left(\omega \backslash \mathscr{B}_{r_{0}}\right)} \leqslant C \varepsilon^{(n+1) \alpha} .
$$

The estimate (5.25) follows from (5.27), (5.28), (5.30) and (5.31). The same technique leads to (5.26) as well. A scaling is needed $(R=r / \varepsilon)$ to recover a domain independent of $\varepsilon$. 
Remark 5.7. Due to estimates (5.25) and (5.26), the outer and corner expansions are unique. Moreover, as the remainders are of the same orders as the first neglected term in the outer and corner expansions, these estimates are optimal. The outer and corner expansions can be seen as Taylor expansions of the exact solution expressed in the $(r, \theta)$ or $(r / \varepsilon, \theta)$ coordinates.

\section{COMPARISON OF THE TWO EXPANSIONS}

In Section 5, starting from the outer and corner (matched) expansions, we were able to build a global asymptotic expansion for the solution $u_{\varepsilon}$ of problem (2.3), see expression (5.14). Using the multiscale technique, we proved in Section 4 another asymptotic expansion, which is also valid in the whole domain $\mathscr{U}_{\varepsilon}$. The global error estimates given in Theorems 5.2 and 4.1 allow to compare these expansions.

Theorem 6.1. The expansions (5.14) and (4.23) compare in the following way:

- The terms $u^{n \alpha}$ and $v^{n \alpha}$ coincide away from the corner point i.e. for $r \geqslant r^{*}$;

- The profiles $U^{n \alpha}$ and $V^{n \alpha}$ coincide in the corner region i.e. for $R \leqslant R^{*} / 2$.

More precisely, we have the identities

$$
\left\{\begin{aligned}
v^{n \alpha}(x) & =u^{n \alpha}(x)-\psi(x) \sum_{p=1}^{n-1} a_{p}^{n} \mathfrak{s}^{-p \alpha}(x), \\
V^{n \alpha}(X) & =U^{n \alpha}(X)-\chi(X) \sum_{p=1}^{n} A_{p}^{n} \mathfrak{s}^{p \alpha}(X) .
\end{aligned}\right.
$$

where the coefficients $a_{p}^{n}$ and $A_{p}^{n}$, are those defined in Section 5.2.

Proof. The first two statements follow directly from the optimal estimates, (5.25), (5.26), (4.23), and (4.24), via localization. To get formulas (6.1), we start from problem (4.19) which defines $V^{n \alpha}$. We set

$$
\begin{aligned}
\widetilde{U}^{n \alpha}(X) & =V^{n \alpha}(X)+\chi(X) \sum_{\ell=0}^{n-1} \mathbf{b}_{n-\ell}^{\ell} \mathfrak{s}^{(n-\ell) \alpha}(X) \\
& =V^{n \alpha}(X)+\chi(X) \sum_{p=1}^{n} \mathbf{b}_{p}^{n-p} \mathfrak{s}^{p \alpha}(X)
\end{aligned}
$$

From the definition of $V^{n \alpha}$ (see (4.19)), $\widetilde{U}^{n \alpha}$ satisfies $\Delta \widetilde{U}^{n \alpha}=0$ in $\Omega$. Hence, one has

$$
\left\{\begin{array}{l}
\widetilde{U}^{n \alpha}-U^{n \alpha} \in C^{\infty}(\Omega), \\
\Delta\left[\widetilde{U}^{n \alpha}-U^{n \alpha}\right]=0 \text { in } \Omega, \\
\widetilde{U}^{n \alpha}(X)-U^{n \alpha}(X)=0 \text { for } R \leqslant R^{*} / 2 .
\end{array}\right.
$$

Since $\widetilde{U}^{n \alpha}-U^{n \alpha}$ is harmonic, it is analytic in $\Omega$. Hence, by unique continuation Theorem, $U^{n \alpha}=\widetilde{U}^{n \alpha}$. Moreover, as $V^{n \alpha}$ is a $\mathcal{O}_{R \rightarrow \infty}(1)$, one has $A_{p}^{n}=\mathbf{b}_{p}^{n-p}$

$$
U^{n \alpha}(X)=V^{n \alpha}(X)+\chi(X) \sum_{p=1}^{n} A_{p}^{n} \mathfrak{s}^{p \alpha}(X) .
$$


The same argumentation can be done for $u^{n \alpha}$.

Remark 6.2. As can be seen in (6.3), another formula linking the two expansions is

$$
\left\{\begin{aligned}
u^{n \alpha}(x) & =v^{n \alpha}(x)+\psi(x) \sum_{p=1}^{n-1} \mathbf{B}_{p}^{n-p} \mathfrak{s}^{-p \alpha}(x), \\
U^{n \alpha}(X) & =V^{n \alpha}(X)+\chi(X) \sum_{p=1}^{n} \mathbf{b}_{p}^{n-p} \mathfrak{s}^{p \alpha}(X) .
\end{aligned}\right.
$$

Moreover, as $A_{p}^{n}=\mathbf{b}_{p}^{n-p}$ and due to the matching condition (5.10), one has

$$
\mathbf{B}_{p}^{\ell}=B_{p}^{\ell} \quad \text { and } \quad \mathbf{b}_{p}^{\ell}=b_{p}^{\ell}, \quad \forall \ell \in \mathbb{N}, \forall p \in \mathbb{N}^{*} .
$$

Remark 6.3. The mechanism to switch from expansion (4.23) to expansion (5.14) consists in using the homogeneity of the singular functions $\mathfrak{s}^{p \alpha}$ to pass them from fast variables into slow variables:

$$
\begin{aligned}
\sum_{\ell=0}^{n} \varepsilon^{\ell \alpha} V^{\ell \alpha}\left(\frac{x}{\varepsilon}\right) & =\sum_{\ell=0}^{n} \varepsilon^{\ell \alpha}\left[U^{\ell \alpha}\left(\frac{x}{\varepsilon}\right)-\chi\left(\frac{x}{\varepsilon}\right) \sum_{p=1}^{\ell} A_{p}^{\ell} \mathfrak{s}^{p \alpha}\left(\frac{x}{\varepsilon}\right)\right] \\
& =\sum_{\ell=0}^{n} \varepsilon^{\ell \alpha} U^{\ell \alpha}\left(\frac{x}{\varepsilon}\right)-\chi\left(\frac{x}{\varepsilon}\right) \sum_{\ell=0}^{n} \sum_{p=1}^{\ell} \varepsilon^{(\ell-p) \alpha} A_{p}^{\ell} \mathfrak{s}^{p \alpha}(x) \\
& =\sum_{\ell=0}^{n} \varepsilon^{\ell \alpha} U^{\ell \alpha}\left(\frac{x}{\varepsilon}\right)-\chi\left(\frac{x}{\varepsilon}\right) \sum_{j=0}^{n} \varepsilon^{j \alpha} \sum_{p=0}^{n-j} \underbrace{A_{p}^{p+\ell} \mathfrak{s}^{p \alpha}(x) .}_{=b_{p}^{\ell}}
\end{aligned}
$$

The second term involves the slow variable and will contribute to the terms $\left(u^{\ell \alpha}\right)$ in the intermediate region.

\section{EXTENSIONS AND GENERALIZATIONS}

The above results can be more or less easily generalized to other situations of interest. For the convenience of the reader, we briefly present a few of them:

(1) Smooth right hand sides $f$ without condition of support,

(2) Neumann boundary conditions,

(3) Small holes and multiple junctions,

(4) Helmholtz operator.

7.1. Smooth data without condition of support. Until now we have assumed that the right hand side $f$ of problem (2.3) is zero in a neighborhood of the limit point $\mathbf{0}$ of the $\varepsilon$-perturbation. This assumption can be relaxed by considering functions $f$ which are restrictions to $\mathscr{U}_{\varepsilon}$ of a $\mathcal{C}^{\infty}$ function $\bar{f}$ defined on a neighborhood of $\cup_{\varepsilon \leqslant \varepsilon_{0}} \mathscr{U}_{\varepsilon}$. In this case we can write

$$
f(x) \underset{r \rightarrow 0}{\simeq} \sum_{q=0}^{+\infty} f^{q}(r, \theta) \quad \text { with } \quad f^{q}(x)=\varepsilon^{q} f^{q}\left(\frac{x}{\varepsilon}\right) .
$$


The asymptotic expansion (7.1) of the right hand-side introduces new terms with integer exponents in the asymptotics of $u_{0}=v^{0}$ as $r \rightarrow 0$ : Instead of the infinite expansion (4.4) we have now

$$
v^{0}(r, \theta) \underset{r \rightarrow 0}{\simeq} \sum_{p=1}^{\infty} \mathbf{b}_{p}^{0} \mathfrak{s}^{p \alpha}(r, \theta)+\sum_{q=1}^{\infty} \mathfrak{T}^{q}(r, \theta),
$$

where $\mathfrak{T}^{q}(r, \theta)$ is a sum of terms of the form $r^{q} \varphi_{0}(\theta)$ and $r^{q} \log r \varphi_{1}(\theta)$ (with $\varphi_{1}=0$ if $\alpha$ is not rational). In turn, these new terms induce new factors with integer powers of $\varepsilon$ in rapid and slow expansions. If $\alpha$ is not a rational number, the expansion of $u_{\varepsilon}$ takes the form, compare with (4.23):

$$
u_{\varepsilon}=\sum_{\substack{p, q \in \mathbb{N} \\ p \alpha+q<s}} \varepsilon^{p \alpha+q}\left(\chi\left(\frac{x}{\varepsilon}\right) v^{p \alpha+q}(x)+\psi(x) V^{p \alpha+q}\left(\frac{x}{\varepsilon}\right)\right)+\mathcal{O}_{\mathrm{H}^{1}}\left(\varepsilon^{s}\right) .
$$

The asymptotics obtained by matched asymptotics expansion contains the same powers of $\varepsilon$ as (7.3). In the case where $\alpha$ is rational, logarithms may appear via the scale $\varepsilon^{p \alpha+q} \log \varepsilon$. For more details, we refer to [19, 3].

7.2. Neumann boundary conditions. Instead of (2.3) let us consider the problem

$$
\text { Find } u_{\varepsilon} \in \mathrm{H}^{1}\left(\mathscr{U}_{\varepsilon}\right) \text { such that } \forall v \in \mathrm{H}^{1}\left(\mathscr{U}_{\varepsilon}\right), \int_{\mathscr{U}_{\varepsilon}} \nabla u_{\varepsilon} \cdot \nabla v \mathrm{~d} x=\int_{\mathscr{U}_{\varepsilon}} f v \mathrm{~d} x \text {. }
$$

Its solvability needs the compatibility condition

$$
\int_{\mathscr{U} \varepsilon} f \mathrm{~d} x=0, \quad \forall \varepsilon<\varepsilon_{0}
$$

Let us assume that $f \equiv 0$ in a neighborhood of 0 and that $\int_{\omega} f \mathrm{~d} x=0$. This implies condition (7.5) for $\varepsilon$ small enough. To ensure uniqueness, we require

$$
\int_{\mathscr{U}_{\varepsilon}} u_{\varepsilon} \mathrm{d} x=0, \quad \forall \varepsilon<\varepsilon_{0} .
$$

The construction of the multiscale expansion for $u_{\varepsilon}$ relies on the solution of variational Neumann problems in $\omega$ and $\Omega$. In the unbounded domain $\Omega$, the variational space $V(\Omega)$ is defined as

$$
\left\{U \in \mathscr{D}^{\prime}(\Omega) ; \quad \nabla U \in \mathrm{L}^{2}(\Omega), \quad(1+R)^{-1}(\log (2+R))^{-1} U \in \mathrm{L}^{2}(\Omega)\right\} .
$$

The bilinear form $(U, V) \longmapsto \int_{\Omega} \nabla U \cdot \nabla V \mathrm{~d} x$ is continuous and coercive on the quotient space $V(\Omega) / \mathbb{R}$, see [2] or [3, Prop. 3.22]. Therefore, like in $\omega$, the solution of the Neumann problem in $\Omega$ with right hand side $F$ requires the compatibility condition $\int_{\Omega} F \mathrm{~d} X=0$. Thus new features have to be taken into account:

(1) Compatibility conditions. The right hand sides which occur during the construction have the form $[\Delta, \psi] \mathfrak{s}^{-p \alpha}$ in $\omega$ and $\left[\Delta_{X}, \chi\right] \mathfrak{s}^{p \alpha}$ in $\Omega$, with the Neumann singularities $\mathfrak{s}^{p \alpha}=\rho^{p \alpha} \cos p \alpha \theta$. Since $\mathfrak{s}^{p \alpha}$ is harmonic, these right hand sides are equal to $\Delta\left(\psi \mathfrak{s}^{-p \alpha}\right)$ and $\Delta_{X}\left(\chi \mathfrak{s}^{p \alpha}\right)$, respectively. The functions $\psi \mathfrak{s}^{-p \alpha}$ and $\chi \mathfrak{s}^{p \alpha}$ satisfying the Neumann boundary condition on $\partial \omega$ and $\partial \Omega$, respectively, we can show that the compatibility conditions are fulfilled for all integer $p \geqslant 1$. 
(2) The role of constants. (i) The asymptotic expansion of $v^{0}$ at $O$ starts with $\mathbf{b}_{0}^{0} \mathfrak{s}^{0}$, which is a constant. The associated problem in fast variables is, $c f$. (4.8)

$$
-\Delta V^{0}=\mathbf{b}_{0}^{0} \Delta_{X} \chi \text { in } \Omega \text { and } \partial_{n} V^{0}=0 \text { on } \partial \Omega .
$$

We choose the solution $V^{0}=\mathbf{b}_{0}^{0}(1-\chi)$. Thus, in particular, $\psi(x) V^{0}\left(\frac{x}{\varepsilon}\right)=V^{0}\left(\frac{x}{\varepsilon}\right)$ : The cut-off by $\psi$ does not introduce any error. Let us notice that the function $\chi\left(\frac{x}{\varepsilon}\right)+\psi(x)(1-\chi)\left(\frac{x}{\varepsilon}\right)$ represents the extension by 1 from $\omega$ to $\mathscr{U}_{\varepsilon}$.

(ii) For problems in $\Omega$ giving $V^{p \alpha}, p \geqslant 1$, we choose the variational solution which tends to 0 as $R \rightarrow \infty$.

(3) The condition for uniqueness. By construction the slow variable terms $v^{p \alpha}$ have a zero integral on $\omega$. Using their asymptotics as $r \rightarrow 0$ we find that

$$
\int_{\mathscr{U}_{\varepsilon}} \chi\left(\frac{x}{\varepsilon}\right) v^{p \alpha}(x) \mathrm{d} x=\beta_{p} \varepsilon^{2}, \quad \beta_{p} \in \mathbb{R} .
$$

For fast variable terms we find

$$
\int_{\mathscr{U}_{\varepsilon}} \psi(x) V^{p \alpha}\left(\frac{x}{\varepsilon}\right) \mathrm{d} x=\beta_{p}^{\prime} \varepsilon^{2}, \quad \beta_{p}^{\prime} \in \mathbb{R} .
$$

We compensate the possible non-zero integral of the multiscale expansion by a series of constant functions - with values $\gamma_{p, n} \in \mathbb{R}, p \in \mathbb{N}, n \in \mathbb{N}^{*}$ - associated with the gauge functions $\varepsilon^{p \alpha+2 n}$. Taking the equality $\int_{\mathscr{U}_{\varepsilon}} \mathrm{d} x=$ meas $\omega+\gamma \varepsilon^{2}$ into account, the $\gamma_{p, n}$ are defined by forcing the formal equality

$$
\sum_{p=0}^{+\infty} \varepsilon^{p \alpha+2}\left(\beta_{p}+\beta_{p}^{\prime}\right)+\left(\operatorname{meas} \omega+\gamma \varepsilon^{2}\right) \sum_{p=0}^{+\infty} \sum_{n=1}^{+\infty} \varepsilon^{2 n+p \alpha} \gamma_{p, n}=0 .
$$

In the end we obtain a multiscale expansion of the form

$$
u_{\varepsilon}=\sum_{\substack{p \in \mathbb{N} \\ p \alpha<s}} \varepsilon^{p \alpha}\left(\chi\left(\frac{x}{\varepsilon}\right) v^{p \alpha}(x)+\psi(x) V^{p \alpha}\left(\frac{x}{\varepsilon}\right)+\sum_{\substack{n \in \mathbb{N}^{*} \\ p \alpha+2 n<s}} \gamma_{p, n} \varepsilon^{2 n}\right)+\mathcal{O}_{\mathrm{H}^{1}}\left(\varepsilon^{s}\right) .
$$

7.3. Small holes. The set $K=\mathbb{R}^{2}$ may also be convenient as a junction set: It allows to consider the case of small holes (or small cracks) of size $\varepsilon$ inside $\mathscr{U}_{\varepsilon}$. This is indeed the first case considered in the book [19, sec. 2.4.1]. Let us consider the Dirichlet case. Then we are in a situation which shares common features with the Dirichlet case investigated in the most part of this paper, and the Neumann case considered above.

Indeed, the limit problem in $\omega$ is uniquely solvable. But the limit problem in $\Omega$ is not coercive on the subspace of $\mathrm{W}_{0}^{1}(\Omega)$ with zero trace on $\partial \Omega$. The correct variational space is the subspace of the space (7.7) with zero trace on $\partial \Omega$. Nevertheless, arguments are slightly different from the Neumann case (like in [3], the asymptotic behavior $\log R$ as $R \rightarrow \infty$ has to be considered). The outcome of the analysis is the appearance of terms $(\log \varepsilon)^{-1}$. Finally, cut-off functions $\chi\left(\frac{x}{\varepsilon}\right)$ and $\psi(x)$ can be simply omitted since $\mathscr{U}_{\varepsilon}$ is a subset of $\omega$ and of $\varepsilon \Omega$. 
7.4. Domains with multiply connected junction sectors. This is the situation where the family of domains $\left(\mathscr{U}_{\varepsilon}\right)$ is defined like in Section 2.1, where we relax the assumption on that the set $K$ is a plane sector. Our results extend to the case where $K$ is a finite disjoint union of plane sectors $K_{1}, \ldots, K_{m}$ with common vertex. Accordingly, we relax the assumption on $\omega$ which is still open and bounded, but can be multiply connected. The unbounded open set $\Omega$ can also be multiply connected. The open sets $\mathscr{U}_{\varepsilon}$ have still to be connected. If $m=2$, this requires that either $\omega$ or $\Omega$ should be connected. Of course, the interesting case occurs when $\Omega$ is connected, see Figure 4.
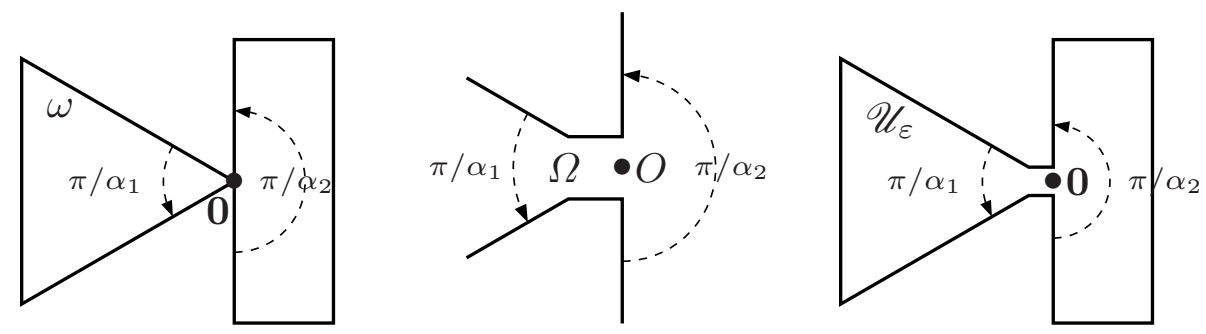

FIGURE 4. Example of domains $\omega, \Omega$ and $\mathscr{U}_{\varepsilon}$ in the multiply connected case $\left(\alpha_{1}=3, \alpha_{2}=1\right)$.

The generalization of our expansions to this situation is straightforward. We denote by $\pi / \alpha_{1}, \ldots, \pi / \alpha_{m}$ the openings of the sectors $K_{1}, \ldots, K_{m}$. The multiscale expansion of $u_{\varepsilon}$ solution of the Dirichlet problem (2.3) with a right hand side $f \equiv 0$ in a neighborhood of $O$ is as follows. For all real number $s>0$ there holds

$$
\begin{aligned}
u_{\varepsilon}= & \sum_{\substack{p_{1}, \ldots, p_{m} \in \mathbb{N} \\
p_{1} \alpha_{1}+\cdots+p_{m} \alpha_{m}<s}} \varepsilon^{p_{1} \alpha_{1}+\cdots+p_{m} \alpha_{m}}\left(\chi\left(\frac{x}{\varepsilon}\right) v^{p_{1} \alpha_{1}+\cdots+p_{m} \alpha_{m}}(x)\right. \\
& \left.+\psi(x) V^{p_{1} \alpha_{1}+\cdots+p_{m} \alpha_{m}}\left(\frac{x}{\varepsilon}\right)\right)+\mathcal{O}_{\mathrm{H}^{1}}\left(\varepsilon^{s}\right) .
\end{aligned}
$$

Here $V^{0}=0$, and $v^{\alpha_{j}}=0$ for $j=1, \ldots, m$. The matched asymptotics exansion is similar.

7.5. Non homogeneous operators : Helmholtz equation. The investigation of the Helmholtz operator in a singularly perturbed domain is of major importance for applications, see [11, 24] for an example of wave propagation in a domain with thin slots. We want to give here the key arguments to derive and justify the matched asymtptotic of the solution of the following model problem, posed in the domain $\mathscr{U}_{\varepsilon}$ defined in (2.1):

$$
\text { Find } u_{\varepsilon} \in \mathrm{H}_{0}^{1}\left(\mathscr{U}_{\varepsilon}\right) \text { such that }-\Delta u_{\varepsilon}-k^{2} u_{\varepsilon}=\left.f\right|_{\mathscr{U}_{\varepsilon}} \text { in } \mathscr{U}_{\varepsilon} \text {, }
$$

where for the sake of simplicity we suppose that

(i) $\alpha$ is not a rational,

(ii) $-k^{2}$ is not an eigenvalue of the Dirichlet Laplacian in the limit domain $\omega$.

The first assumption avoids the occurrence of a logarithmic gauge function in the asymptotic expansions. The second leads to a well-posed limit problem. 
This situation is rather more technical since this operator is not self-similar :

$$
\Delta_{x}+k^{2}=\frac{1}{\varepsilon^{2}}\left(\Delta_{X}+\varepsilon^{2} k^{2}\right) .
$$

A second difficulty comes from the loss of coercivity. The proofs of existence and convergence need then to be modified, see for example [19, Ch.4] and [11, 24].

Some preliminaries on super-variational problems. According to a common usage, we denote by $J_{p \alpha}$ and $Y_{p \alpha}$ the Bessel function of first and second kind of order $p \alpha$, respectively, see for instance [14].

Proposition 7.1. Under condition (ii), for any finite sequence $\left(a_{p}\right)_{1 \leqslant p \leqslant P}$ of real numbers, there exists a unique solution u to the "super-variational problem"

$$
\left\{\begin{array}{l}
\text { Find } u \in V_{\mathrm{loc}, 0}(\omega) \text { such that } \\
\Delta u+k^{2} u=0 \text { in } \omega \text { and } u(x)=\sum_{p=1}^{P} a_{p} Y_{p \alpha}(k r) \sin (p \alpha \theta)+\mathcal{O}_{r \rightarrow 0}(1) .
\end{array}\right.
$$

In the neighborhood of 0 , this solution can be expanded as follows

$$
u(x) \underset{r \rightarrow 0}{\simeq} \sum_{p=1}^{P} a_{p} Y_{p \alpha}(k r) \sin (p \alpha \theta)+\sum_{p=1}^{+\infty} b_{p} J_{p \alpha}(k r) \sin (p \alpha \theta) .
$$

Let $J_{p \alpha, \ell}$ and $Y_{p \alpha, \ell}$ be the coefficients of the generalized Taylor series of the Bessel functions $J_{p \alpha}$ and $Y_{p \alpha}$ (the coefficients for odd $\ell$ are all zero):

$$
J_{p \alpha}(z)=z^{p \alpha} \sum_{\ell \in \mathbb{N}} J_{p \alpha, \ell} z^{\ell} \quad \text { and } \quad Y_{p \alpha}(z)=\frac{1}{z^{p \alpha}} \sum_{\ell \in \mathbb{N}} Y_{p \alpha, \ell} z^{\ell}
$$

Proposition 7.2. Under condition ( $i)$, for any finite sequence $\left(A_{p}^{m}\right)_{1 \leqslant p \leqslant P, 0 \leqslant m \leqslant M}$ of real numbers, there exists a unique solution $\left(U^{m}\right)_{0 \leqslant m \leqslant M}$ of the "super-variational system"

$$
\left\{\begin{array}{c}
\text { For } m=0, \ldots, M, \text { find } U^{m} \in V_{\mathrm{loc}, \infty}(\Omega) \text { such that } \\
\Delta U^{m}+k^{2} U^{m-2}=0 \text { in } \Omega, \quad\left(\text { with convention } U^{m}=0 \text { if } m<0\right) \\
U^{m}(X)=\sum_{p=1}^{P} \sum_{\ell=0}^{m} A_{p}^{m-\ell} J_{p \alpha, \ell}(k R)^{p \alpha+\ell} \sin (p \alpha \theta) \\
+\sum_{p=1}^{+\infty} \sum_{\ell=1}^{m} B_{p}^{m-\ell} Y_{p \alpha, \ell}(k R)^{-p \alpha+\ell} \sin (p \alpha \theta)+\mathcal{O}_{R \rightarrow \infty}(1),
\end{array}\right.
$$

where $\left(B_{p}^{m}\right)_{0 \leqslant p \leqslant+\infty, 0 \leqslant m \leqslant M}$ are the coefficents such that in the neighborhood of infinity there holds

$$
\begin{aligned}
U^{m}(X) \underset{R \rightarrow+\infty}{\simeq} & \sum_{p=1}^{P} \sum_{\ell=0}^{m} A_{p}^{m-\ell} J_{p \alpha, \ell}(k R)^{p \alpha+\ell} \sin (p \alpha \theta) \\
& +\sum_{p=1}^{+\infty} \sum_{\ell=0}^{m} B_{p}^{m-\ell} Y_{p \alpha, \ell}(k R)^{-p \alpha+\ell} \sin (p \alpha \theta) .
\end{aligned}
$$


Remark 7.3. If condition $(i)$ is not satisfied, the expansions of $J_{p \alpha}$ and $Y_{p \alpha}$ do not only include terms of the form $r^{\mu}$ but also terms of the form $r^{\mu}(\ln r)^{\nu}$.

Definition of the matched asymtptotic expansions. The gauge functions appearing in the asymptotic expansions of $u_{\varepsilon}$ are of the form $\varepsilon^{m+n \alpha}$, ie. we look for the two asymptotic expansions with the form

$$
u_{\varepsilon}(x) \simeq \sum_{(m, n) \in \mathbb{N}^{2}} \varepsilon^{m+n \alpha} U^{m, n}\left(\frac{x}{\varepsilon}\right) \quad \text { and } \quad u_{\varepsilon}(x) \simeq \sum_{(m, n) \in \mathbb{N}^{2}} \varepsilon^{m+n \alpha} u^{m, n}(x) .
$$

The coefficients of these expansions can be defined hierarchically as the unique solutions of the coupled problem

$$
\left\{\begin{array}{l}
\text { Find } u^{m, n} \in V_{\mathrm{loc}, 0}(\omega) \text { such that } \\
\Delta u^{m, n}+k^{2} u^{m, n}=0 \text { in } \omega, \quad(\text { or }-f \text { if } m=n=0) \\
u^{m, n}-\sum_{p=1}^{n} a_{p}^{m, n} Y_{p \alpha}(k r) \sin (p \alpha \theta)=\mathcal{O}_{r \rightarrow 0}(1),
\end{array}\right.
$$

$$
\left\{\begin{array}{c}
\text { Find } U^{m, n} \in V_{\mathrm{loc}, \infty}(\Omega) \text { such that } \\
\begin{array}{r}
\Delta U^{m, n}+k^{2} U^{m-2, n}=0 \text { in } \Omega, \quad\left(\text { with } U^{m, n}=0 \text { if } m<0\right) \\
U^{m, n}(X)-\sum_{p=1}^{n} \sum_{\ell=0}^{m} A_{p}^{m-\ell, n} J_{p \alpha, \ell}(k R)^{p \alpha+\ell} \sin (p \alpha \theta) \\
-\sum_{p=1}^{+\infty} \sum_{\ell=1}^{m} B_{p}^{m-\ell, n} Y_{p \alpha, \ell}(k R)^{-p \alpha+\ell} \sin (p \alpha \theta)=\mathcal{O}_{R \rightarrow \infty}(1),
\end{array}
\end{array}\right.
$$

together with the matching conditions

$$
a_{p}^{m, n}=B_{p}^{m, n-p} \quad \text { and } \quad A_{p}^{m, n}=b_{p}^{m, n-p} \quad \text { if } 1 \leqslant p \leqslant n,
$$

where the coefficients $b_{p}^{m, n}$ are defined through the sub-variational expansion of $u^{m, n}$ :

$$
u^{m, n}(x) \underset{r \rightarrow 0}{\simeq} \sum_{p=1}^{n} a_{p}^{m, n} Y_{p \alpha}(k r) \sin (p \alpha \theta)+\sum_{p=1}^{+\infty} b_{p}^{m, n} J_{p \alpha}(k r) \sin (p \alpha \theta) .
$$

\section{Error Estimates.}

Theorem 7.4. Let $I_{N}$ be the set of indices corresponding to gauge functions of order lower than $N$

$$
I_{N}=\left\{(m, n) \in \mathbb{N}^{2}: m+n \alpha \leqslant N\right\} .
$$

The global approximation is defined by

$$
\left\{\begin{aligned}
\widehat{u}_{N}^{\varepsilon}(x)= & \varphi\left(\frac{r}{\eta(\varepsilon)}\right) \sum_{(m, n) \in I_{N}} \varepsilon^{m+n \alpha} u^{m, n}(x) \\
& +\left(1-\varphi\left(\frac{r}{\eta(\varepsilon)}\right)\right) \sum_{(m, n) \in I_{N}} \varepsilon^{m+n \alpha} U^{m, n}\left(\frac{x}{\varepsilon}\right),
\end{aligned}\right.
$$


where $\varphi$ is a smooth cut-off function with $\varphi(\rho)=0$ for $\rho<1$ and $\varphi(\rho)=1$ for $\rho>2$ and $\eta$ is smooth and satisfies

$$
\lim _{\varepsilon \rightarrow 0} \eta(\varepsilon)=0 \quad \text { and } \quad \lim _{\varepsilon \rightarrow 0} \frac{\eta(\varepsilon)}{\varepsilon}=+\infty .
$$

There exists a constant $C$ such that

$$
\left\|u_{\varepsilon}-\widehat{u}_{N}^{\varepsilon}\right\|_{\mathrm{H}^{1}(\mathscr{U} \varepsilon)} \leqslant C\left[(\eta(\varepsilon))^{N}+\left(\frac{\varepsilon}{\eta(\varepsilon)}\right)^{N}\right] .
$$

\section{CONCLUSION: A PRACTICAL TWO-TERM EXPANSION}

In order to investigate the influence of singular perturbations of the domain on a local functional $\varphi_{\varepsilon}$ acting over the solution $u_{\varepsilon}$, it is valuable to use a compound version of the asymptotic expansion, in between multiscale and matched asymptotic expansions.

8.1. Compound expansion. Indeed, using (2.9) and the relation (2.11) between the profiles $V^{\alpha}$ and $U^{\alpha}$, we get

$$
u_{\varepsilon}=\chi\left(\frac{x}{\varepsilon}\right) u_{0}(x)+\psi(x) \varepsilon^{\alpha}\left[U^{\alpha}\left(\frac{x}{\varepsilon}\right)-\chi\left(\frac{x}{\varepsilon}\right) A \mathfrak{s}^{\alpha}\left(\frac{x}{\varepsilon}\right)\right]+\mathcal{O}_{\mathrm{H}^{1}}\left(\varepsilon^{2 \alpha}\right),
$$

which can be written, thanks to the homogeneity of the singular function $\mathfrak{s}^{\alpha}$

$$
u_{\varepsilon}=\chi\left(\frac{x}{\varepsilon}\right)\left[u_{0}(x)-A \psi(x) \mathfrak{s}^{\alpha}(x)\right]+\psi(x) \varepsilon^{\alpha} U^{\alpha}\left(\frac{x}{\varepsilon}\right)+\mathcal{O}_{\mathrm{H}^{1}}\left(\varepsilon^{2 \alpha}\right) .
$$

Let us introduce the first "canonical" profile $U_{\Omega}^{\alpha}$ as the solution of the super-variational Dirichlet problem on $\Omega$

$$
\left\{\begin{array}{l}
\text { Find } U_{\Omega}^{\alpha} \in V_{\mathrm{loc}, \infty}(\Omega) \text { such that } \\
\quad \Delta U_{\Omega}^{\alpha}=0 \text { in } \Omega \text { and } U_{\Omega}^{\alpha}-\mathfrak{s}^{\alpha}=\mathcal{O}_{R \rightarrow \infty}(1) .
\end{array}\right.
$$

We have $U^{\alpha}=A U_{\Omega}^{\alpha}$ and, hence

$$
u_{\varepsilon}=\chi\left(\frac{x}{\varepsilon}\right)\left[u_{0}(x)-A \psi(x) \mathfrak{s}^{\alpha}(x)\right]+\psi(x) \varepsilon^{\alpha} A U_{\Omega}^{\alpha}\left(\frac{x}{\varepsilon}\right)+\mathcal{O}_{\mathrm{H}^{1}}\left(\varepsilon^{2 \alpha}\right) .
$$

In (8.2), only canonical objects are involved: the limit term $u_{0}$, its first singularity coefficient $A$, and the first profile $U_{\Omega}^{\alpha}$. The contribution near the corner is fully contained in the profile $A U_{\Omega}^{\alpha}$, whereas the "far-field" information is carried out by $u_{0}-A \psi \mathfrak{s}^{\alpha}$, corresponding to the limit term without its first singularity. In a sense, the strongest singularity of $u_{0}$ is "chopped off" for $\varepsilon>0$ via the cut-off $\chi\left(\frac{x}{\varepsilon}\right)$, and is replaced with the profile $A U_{\Omega}^{\alpha}$, which connects the local geometry around $O$ with the plane sector of opening $\alpha$ at infinity.

8.2. Application: asymptotics of coefficients of singularities. An interesting application of expansion (8.2) is the determination of Stress Intensity Factors at the tip of a short crack emanating from a sharp of a rounded V-notch, see [16]. More generally, the question is the determination of the asymptotic behavior of the coefficients of singularities of $u_{\varepsilon}$ associated with the corners (or cracks) of the domain $\mathscr{U}_{\varepsilon}$ inside its perturbed region. The functional $\varphi_{\varepsilon}\left(u_{\varepsilon}\right)$ is then defined as the value of this coefficient of singularity, corresponding to a corner whose position depends on $\varepsilon$.

Indeed, to each corner point (or crack tip) $d$ of the perturbation pattern $\Omega$ corresponds a corner point (or crack tip) $d_{\varepsilon}$ of the perturbed domain $\mathscr{U}_{\varepsilon}$. In Figure 5, two such points are involved, both associated with angle $2 \pi$. 

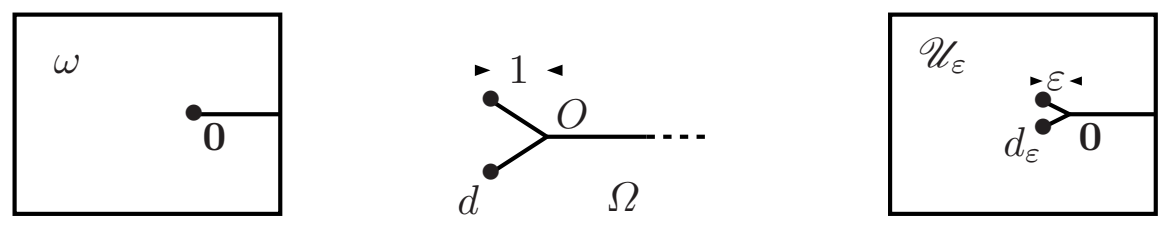

FIGURE 5. Crack tips: Domains $\omega, \Omega$ and $\mathscr{U}_{\varepsilon}$.

The solution $u_{\varepsilon}$ of the Laplace-Dirichlet problem (2.3) is singular at point $d_{\varepsilon}$, with the following first order approximation

$$
u_{\varepsilon}(x)=\gamma_{\varepsilon} r_{\varepsilon}^{\mu} \sin \left(\mu \theta_{\varepsilon}\right)+\mathcal{O}_{\varepsilon}\left(r_{\varepsilon}^{\mu}\right), \quad \text { as } r_{\varepsilon} \rightarrow 0,
$$

where $\left(r_{\varepsilon}, \theta_{\varepsilon}\right)$ denote the polar coordinates around $d_{\varepsilon}$. The exponent $\mu$ is the singular exponent corresponding to $d_{\varepsilon}(\mu=\pi / \vartheta$ for a corner of opening $\vartheta, \mu=1 / 2$ for a crack). The functional $\varphi_{\varepsilon}$ is defined as

$$
\varphi_{\varepsilon}\left(u_{\varepsilon}\right)=\gamma_{\varepsilon}
$$

Our results allow to give an asymptotic expansion of the singular coefficient $\gamma_{\varepsilon}$ as $\varepsilon \rightarrow 0$ : we still denote by $\alpha$ the singular exponent associated with the limit problem in $\omega$. Using (8.2), we get

$$
u_{\varepsilon}(x)=\varepsilon^{\alpha} A U_{\Omega}^{\alpha}\left(\frac{x}{\varepsilon}\right)+\text { higher order profiles, if }|x| \leqslant \varepsilon r_{*} .
$$

But the first canonical profile $U_{\Omega}^{\alpha}$ has a singularity at point $d$, associated with exponent $\mu$

$$
U_{\Omega}^{\alpha}(X)=\Gamma R_{d}^{\mu} \sin \left(\mu \Theta_{d}\right)+\mathcal{O}\left(R_{d}^{\mu}\right), \quad \text { as } R_{d} \rightarrow 0,
$$

where $\left(R_{d}, \Theta_{d}\right)$ are the polar coordinates around point $d$. We have the relation

$$
r_{\varepsilon}=\varepsilon R_{d} \quad \text { and } \quad \theta_{\varepsilon}=\Theta_{d} .
$$

Back to the variable $x$, relations (8.4) to (8.6) lead to

$$
u_{\varepsilon}(x)=\varepsilon^{\alpha-\mu} A \Gamma r_{\varepsilon}^{\mu} \sin \left(\mu \theta_{\varepsilon}\right)+\mathcal{O}\left(\varepsilon^{\alpha-\mu} r_{\varepsilon}^{\mu}\right), \quad \text { if }|x| \leqslant \varepsilon r_{*} .
$$

Putting (8.3) and (8.7) together, we obtain the expression of the singular coefficient $\gamma_{\varepsilon}$

$$
\gamma_{\varepsilon}=\varepsilon^{\alpha-\mu} A \Gamma+\mathcal{O}\left(\varepsilon^{\alpha-\mu}\right) .
$$

It is worth noticing that a coefficient associated with a stronger singularity than the limit singularity $(\mu<\alpha)$ will go to 0 whereas it will blow up to infinity for weaker singularities. It has to be linked to the appearance of singularities discussed above. In the case of Figure 5 we have $\alpha=1 / 2$ and $\mu=1 / 2$. The coefficient associated with the $\mathrm{v}$-notch cracks is a $\mathcal{O}(1)$.

The above analysis also applies in the framework of elasticity, and is the foundation of the investigation in [16]. We stress that a rigorous derivation of (8.8) with an optimal estimate for the remainder requires more effort in studying the singular-regular expansion of $u_{\varepsilon}$.

Expansion (8.2) could also be used to investigate the asymptotic behavior of other local functionals $\varphi_{\varepsilon}\left(u_{\varepsilon}\right)$ relating, for example, to the maximal values of the stress tensor in elasticity. 


\section{REFERENCES}

[1] S. Agmon. Lectures on elliptic boundary value problems. Prepared for publication by B. Frank Jones, Jr. with the assistance of George W. Batten, Jr. Van Nostrand Mathematical Studies, No. 2. D. Van Nostrand Co., Inc., Princeton, N.J.-Toronto-London 1965.

[2] C. Amrouche, V. Girault, J. Giroire. Dirichlet and Neumann exterior problems for the $n$-dimensional Laplace operator: an approach in weighted Sobolev spaces. J. Math. Pures Appl. (9) 76(1) (1997) 55-81.

[3] G. Caloz, M. Costabel, M. Dauge, G. Vial. Asymptotic expansion of the solution of an interface problem in a polygonal domain with thin layer. to appear in Asymptotic Analysis (2006).

[4] M. Costabel, M. Dauge. A singularly perturbed mixed boundary value problem. Comm. Partial Differential Equations 21 (1996) 1919-1949.

[5] M. Costabel, M. Dauge, Z. Yosibash. A quasi-dual function method for extracting edge stress intensity functions. SIAM J. Math. Anal. 35(5) (2004) 1177-1202 (electronic).

[6] M. DAuge. Elliptic Boundary Value Problems in Corner Domains - Smoothness and Asymptotics of Solutions. Lecture Notes in Mathematics, Vol. 1341. Springer-Verlag, Berlin 1988.

[7] M. Dauge, S. Nicaise, M. Bourlard, J. M.-S. Lubuma. Coefficients des singularités pour des problèmes aux limites elliptiques sur un domaine à points coniques. I. Résultats généraux pour le problème de Dirichlet. RAIRO Modél. Math. Anal. Numér. 24(1) (1990) $27-52$.

[8] R. R. GADYL'SHIN. Surface potentials and the method of matching asymptotic expansions in the Helmholtz resonator problem. Algebra i Analiz 4(2) (1992) 88-115.

[9] P. GRISVARD. Boundary value problems in non-smooth domains. Pitman, London 1985.

[10] A. IL'LIN. Matching of asymptotic expansions of solutions of boundary value problems. Translations of Mathematical Monographs (1992).

[11] P. Joly, S. TordeuX. Matching of asymptotic expansions for wave propagation in media with thin slots i: The asymptotic expansion. Multiscale Modeling and Simulation: A SIAM Interdisciplinary Journal 5(1) (2006) 304-336.

[12] V. A. KONDRAT'EV. Boundary value problems for elliptic equations in domains with conical or angular points. Trans. Moscow Math. Soc. 16 (1967) 227-313.

[13] V. A. Kozlov, V. G. MaZ’YA, J. Rossmann. Elliptic boundary value problems in domains with point singularities. Mathematical Surveys and Monographs, 52. American Mathematical Society, Providence, RI 1997.

[14] N. N. Lebedev. Special functions and their applications. Dover Publications Inc., New York 1972. Revised edition, translated from the Russian and edited by Richard A. Silverman, Unabridged and corrected republication.

[15] D. Leguillon, E. SANCheZ-Palencia. Computation of singular solutions in elliptic problems and elasticity. Masson, Paris 1987. 
[16] D. Leguillon, Z. Yosibash. Crack onset at a v-notch. Influence of the notch tip radius. Int. Jour. Fracture 122 (2003) 1-21.

[17] V. G. Maz'Ja, S. A. Nazarov, B. A. Plamenevskil̆. Asymptotic behavior of the solutions of elliptic boundary value problems in the case of variation of the domain near conic points. Dokl. Akad. Nauk SSSR 249(1) (1979) 94-96.

[18] V. G. MaZ'Ya, S. A. NAZARov, B. A. Plamenevskil. On the asymptotic behavior of solutions of elliptic boundary value problems with irregular perturbations of the domain. volume 8 of Probl. Mat. Anal., pages 72-153, 222-223. Leningrad. Univ., Leningrad 1981.

[19] V. G. Maz'ya, S. A. Nazarov, B. A. Plamenevskij. Asymptotic theory of elliptic boundary value problems in singularly perturbed domains. Birkhäuser, Berlin 2000.

[20] V. G. MaZ'Ya, B. A. Plamenevskit. Estimates in $L^{p}$ and in Hölder classes and the Miranda-Agmon maximum principle for solutions of elliptic boundary value problems in domains with singular points on the boundary. Amer. Math. Soc. Transl. (2) 123 (1984) 1-56.

[21] V. G. MaZ'Ya, B. A. PlamenevskiI. On the coefficients in the asymptotic of solutions of the elliptic boundary problem in domains with conical points. Amer. Math. Soc. Trans. (2) 123 (1984) 57-88.

[22] S. A. Nazarov, B. A. Plamenevsky. Elliptic problems in domains with piecewise smooth boundaries, volume 13 of de Gruyter Expositions in Mathematics. Walter de Gruyter \& Co., Berlin 1994.

[23] O. A. Oleinik, A. S. Shamaev, G. A. Yosifian. Mathematical Problems in Elasticity and Homogenization. Studies in mathematics and its applications. North-Holland, Amsterdam 1992.

[24] S. Tordeux. Méthodes asymptotiques pour la propagation des ondes dans les milieux comportant des fentes. Thèse de doctorat. (2004).

[25] M. VanDyke. Perturbation methods in fluid mechanics. The Parabolic Press. (1975).

[26] G. Vial. Analyse multi-échelle et conditions aux limites approchées pour un problème de couche mince dans un domaine à coin. Thèse de doctorat 2840, Université de Rennes I, IRMAR 2003.

\section{ADDRESSES}

IRMaR, Université de Rennes 1, Campus de Beaulieu, 35042 Rennes Cedex, France

E-mail address: monique. dauge@univ-rennes1.fr

Institut de Mathématiques de Toulouse, INSA-Toulouse, 31077 Toulouse, France

E-mail address: sebastien.tordeux@insa-toulouse.fr

IRMAR, ENS Cachan Bretagne, CNRS, UEB, 35170 Bruz, France

E-mail address: gvial@bretagne.ens-cachan.fr 